\title{
ANATOMÍA FOLIAR EN ESPECIES DE CAPSICUM (SOLANACEAE) DE DIFERENTES AMBIENTES BIOGEOGRÁFICOS SUDAMERICANOS
}

\author{
MARÍA VIRGINIA PALCHETTI'1, GLORIA ESTELA BARBOZA² y MARÍA TERESA COSA1,2
}

\begin{abstract}
Summary: Leaf anatomy in Capsicum species (Solanaceae) from different biogeographic South American environments. In this work, the leaf anatomy of 13 Capsicum species growing in different Biogeographic Provinces was studied. A comparative analysis of the leaf epidermis and its internal structure was carried out in order to find differences and/or similarities among the species and the presence/absence of mesophytic and/or xerophytic characters according to their environment conditions. The density of epidermal cells, stomata, trichomes and stomatal index were statistically analyzed. The results showed that all the species have similar characters; however, there are some unique features that characterize the species as hypostomatic leaves, presence and types of trichomes, presence or absence of crystals and its variable disposition, fibers in main vein, and probable presence of tannins. Regarding the presence of xeromorphic or mesomorphic characters, the differences were not significant among the species from different environments except for the amphistomatic leaves and the abundance of crystals, and trichomes in species from dry environments. We conclude that the similarity of the leaf epidermal variables and the internal structure observed in the Capsicum species are responding more to a grouping according to their phylogenetic relationships than to the environmental conditions where they live.
\end{abstract}

Key words: adaptations, anatomical features, Capsiceae, chili pepper, taxonomy.

Resumen: Se estudió la anatomía foliar en 13 especies de Capsicum L. que crecen en diferentes Provincias Biogeográficas. Se realizó un análisis comparativo de la epidermis foliar y de la estructura interna de lámina y pecíolo para hallar diferencias y/o similitudes entre las especies y la presencia/ausencia de caracteres mesomórficos/xeromórficos según el ambiente que habiten. La densidad de las células epidérmicas propiamente dichas, de los estomas, de los diferentes tipos de tricomas y el índice estomático fueron estadísticamente analizados. Los resultados indican que todas las especies presentan características similares en sus hojas. Sin embargo, en determinadas especies se observaron algunas particularidades, tales como hojas hipostomáticas, presencia y tipos de tricomas, presencia, disposición y tipo de cristales, fibras rodeando la vena media y probable presencia de taninos. No se encontraron diferencias significativas entre especies que habitan distintos ambientes, excepto aquellas especies procedentes de ambientes secos con hojas anfistomáticas y abundancia de cristales y tricomas. Se concluye que las similitudes de las variables epidérmicas y de la estructura interna de la hoja, observadas en las especies de Capsicum, estarían respondiendo a un agrupamiento según sus relaciones filogenéticas más que a las condiciones ambientales donde habitan.

Palabras clave: adaptaciones, Capsiceae, características anatómicas, pimiento, taxonomía.

\section{INTRODUCCIÓN}

Capsicum L. (tribu Capsiceae, Solanaceae) es un género netamente americano de ca. 32 especies (Barboza et al., 2011). Se reconoce fácilmente por la

${ }^{1}$ Laboratorio de Morfología Vegetal, FCEFyN. Universidad Nacional de Córdoba, Argentina.

2 IMBIV, CONICET y Universidad Nacional de Córdoba, Argentina. Casilla de Correo 495, 5000. Córdoba, Argentina.

E-mail: palchettimv@hotmail.com morfología del cáliz mútico (sin lóbulos), generalmente con apéndices o dientes notables que nacen por debajo del borde superior. El género es de reconocida importancia económica; comprende a los "ajíes", "chiles", "guindillas" o "pimientos", cuyos frutos contienen capsaicina, sustancia irritante, picante o acre, responsable del sabor y picor característicos que presentan (Hunziker, 2001). Para el género son reconocidas cinco especies domesticadas: $C$. annuum L., C. baccatum L., $C$. chinense Jacq., C. frutescens L. y C. pubescens Ruiz 
\& Pav. (Buckenhüskes, 2003; Moscone et al., 2007; Barceloux, 2008).

Sobre la base de diferentes análisis moleculares, filogenéticamente, el género Capsicum L. ha sido ubicado en el clado Capsiceae junto con Lycianthes Hassl., su pariente más cercano (Olmstead et al., 2008; Särkinen et al., 2013). En los últimos años, diferentes estudios citogenéticos y análisis moleculares han facilitado la caracterización y el establecimiento de grupos taxonómicos de Capsicum. Sin embargo, no se ha propuesto ninguna clasificación infragenérica y el número exacto de especies sigue siendo incierto (Barboza et al., 2011).

Las especies de Capsicum son nativas de Sur y Centro América. Brasil es el país con la mayor concentración de especies (Barboza \& Bianchetti, 2005; Barboza et al., 2011), especialmente en la zona costera del sudeste (Mata Atlántica). Sólo unos pocos taxones han sido registrados en el norte y nordeste de este país, zona con una rica flora en endemismos (Giulietti et al., 2002; Queiroz, 2006).

Otro grupo importante de especies crece desde el norte de Sudamérica hasta el centro de Argentina. En nuestro país, se encuentran en ambientes con clima húmedo de las Provincias Biogeográficas Paranaense y de las Yungas o en zonas más secas de la Provincia Biogeográfica Chaqueña (Cabrera \& Willink, 1980).

Dado que las especies de este género crecen en distintas regiones biogeográficas, algunas de ellas presentan adaptaciones al ambiente en el que viven (Lleras, 1997).

Una adaptación es una característica que aumenta la supervivencia o la reproducción de los organismos que la poseen, en relación a los estados alternativos del carácter y se desarrolla mediante selección natural. También se refiere al proceso por el cual una especie se adapta a algún rasgo de su medio ambiente a través del cambio en una característica que afecte su supervivencia o reproducción (Futuyma, 2009). Las adaptaciones permiten a las plantas sobrevivir en condiciones extremas de frío, calor, humedad, sequía y deficiencia de minerales del suelo. El estrés juega un rol importante en la evolución adaptativa y el rango de temperatura y disponibilidad de agua están entre los factores abióticos más importantes que determinan el grado de especificidad de la adaptación (Fahn \& Cutler, 1992).

Las adaptaciones en la estructura de las plantas, relacionadas con factores ambientales, se manifiestan particularmente en la morfología y anatomía de la hoja (Cosa, 1991; Fahn \& Cutler, 1992; Cosa et al., 2002; Delbón et al., 2007). Este órgano ha sido considerado el más variable de la planta y reconocido como indicador de condiciones ambientales (Dickison, 2000).

El presente estudio plantea el análisis comparativo de la estructura foliar en especies de Capsicum que habitan regiones biogeográficas contrastantes, caracterizadas por tener diferentes rangos de temperatura y humedad y diferentes gradientes altitudinales, con el fin de comprender sus estrategias adaptativas a diversos ambientes y valorar si tiene incidencia en la sistemática del grupo.

\section{MATERIALES Y MÉTOdOS}

\section{Material estudiado}

Capsicum baccatum L. ARGENTINA. Prov. Misiones: Dpto. Capital, Posadas, cultivado en la vereda sobre Avda. San Martín, 7-X-2011, Barboza \& Cosa 3055 (CORD). Prov. Jujuy: Dpto. Ledesma, Parque Nacional Calilegua, 6-IV2007, Barboza 1921 bis (CORD). Ldor. Gral. San Martín, Finca Yuto, 21-III-2005, Barboza et al. 1274 (CORD).

Capsicum caatingae Barboza \& Agra. Brasil. Est. Bahia: Miguel Calmon, Piemonte da Diamantina, 525 msm, 23-XII-2006, Guedes et al. 13245 \& 13297 (ALCB). Serra Preta, Faz. Santa Clara Nova, 262 msm, 2-IV-2009, Agra et al. 7081 (JPB). Itatim, Morro do Quixaba, 273 msm, 3-IV2009, Agra et al. 7085 (CORD, JPB). Feira de Santana, Distrito de São José de Ytapororoca, Serra de São José, Bautista \& Pinto 1023 (CORD).

Capsicum cardenasii Heiser \& P.G. Sm. Estados Unidos de América. Cult. at IU greenhouse (seeds from La Paz, Bolivia), 21-III-1960, Heiser C271 (CORD, US). BOLIVIA. Dpto. La Paz: Prov. Loayza, 2500 msm, 5-IV-1994, Beck 130 PG94 (CORD, LPB).

Capsicum chacoense Hunz. ARGENTINA. Prov. Córdoba: Dpto. Ischilín, Estancia Los Ruices, \pm 930 msm, 15-I-2012, Cosa \& Palchetti 399 (CORD). Dpto. Sobremonte, a $6 \mathrm{~km}$ al Oeste de la Iglesia de San Francisco del Chañar, Subils 3873 (CORD). Prov. Chaco: Dpto. $1^{\circ}$ de Mayo, Colonia Benítez, 15-IV-1944, Schulz 4272 (CORD).

Capsicum cornutum Hunz. Brasil. Est. São Paulo: Munic. Santo André, Paranapiacaba, 775 msm, 


\section{V. Palchetti et al. - Anatomía foliar en Capsicum}

25-IX-2010, Barboza \& Cosa 2525 (CORD). Reserva Biológica do Alto da Serra de Paranapiacaba, 800 msm, 24-IX-2010, Barboza \& Cosa 2517 (CORD). Serra do Mar, Estación Biológica de Paranapiacaba, 850 msm, 1-XII-67, Hunziker 19557 (CORD).

Capsicum eximium Hunz. ARGENTINA. Prov. Salta: Dpto. Capital, Sierra de Vélez, Cerro de la ermita, 1300-1400 msm, 15-I-2005, Novara et al. 12206 (CORD, MCNS). Ciudad, cultivado en jardín particular Sr. E. Lusvarghi (Barrio Tres Cerritos), 20-I-2007, Barboza 1919 (CORD). Dpto. Gral. Güemes, Güemes, 1998, Neumann s.n. (CORD 00019083).

Capsicum flexuosum Sendtn. Argentina. Prov. Corrientes: Dpto. Santo Tomé, Garruchos, Estancia San Juan Bautista, 100 msm, 7-IV-2005, Barboza et al. 1487 (CORD). Prov. Misiones: Dpto. Candelaria, Bonpland, 3-VII-2004, Daviña \& Honfi 599 (MNES). Dpto. Gral. M. Belgrano, Parque Provincial Urugua-í, $360 \mathrm{msm}, 17-$ II1996, Morrone et al. 9312 (CORD). Brasil. Est. Paraná: Cataratas del Iguazú, Hunziker 20232 (CORD).

Capsicum friburgense Bianch. \& Barboza. BRASIL. Est. Rio de Janeiro: Munic. Nova Friburgo, subindo o Morro da Caledônia, 1800 msm, 6-IV1986, Bianchetti et al. 391 \& 393 (CEN; CORD). Pico alto de Caledônia, $\pm 1880 \mathrm{msm}, 16-\mathrm{V}-88$, Hunziker 25267 (CORD).

Capsicum geminifolium Hunz. Colombia. Dpto. Valle del Cauca: filo de la Cordillera Occidental al sur de Las Brisas, 1850 msm, 27-X-1946, Cuatrecasas 22665 (CORD, F, US). Hoya del río Dígua, ca. 1200 msm, 27-29-III-1947, Cuatrecasas 23996 (CORD, F). Dpto. Quindio: Munic. Circasia, vereda Membrillal, $1700 \mathrm{msm}$, 13-II-2010, Beltrán 085 (CORD).

Capsicum hunzikerianum Barboza \& Bianch. BRASIL. Est. São Paulo: a unos $17 \mathrm{~km}$ al sur de Bananal, $1150 \mathrm{msm}, 26-\mathrm{II}-2006$, Barboza et al. 1648 \& 1649 (CORD).

Capsicum longidentatum Agra \& Barboza. Brasil. Est. Bahia: Munic. Itatim, Cerca de Pedra Resoles, 295 msm, 3-IV-2009, Agra et al. 7083 (CORD, JPB). Morro do Quixaba, $273 \mathrm{msm}$, 3IV-2009, Agra \& Barboza 7086 (CORD, JPB). Est. Pernambuco: Pernambuco, Mirandiba, Serra do Tigre, 495 msm, 18-IV-2007, Pinheiro 253 (CORD, JPB, UFP).

Capsicum recurvatum Witasek. Brasil. Est. Santa Catharina: Munic. Presidente Nereu, Estrada Madeireira, 770 msm, 27-IV-1985, Hunziker
24998 (CORD). Estrada a Sabiá, 660 msm, 27IV-1985, Hunziker 25001 (CORD, ICN). Munic. Cunha, Camino entre Cunha y el cerro Pedra Marcela, 1650 msm, 13-IV-1986, Hunziker 25184 (CORD).

Capsicum villosum Sendtn. BRASIL. Est. Minas Gerais: Munic. Itamonte, BR-354, Km 778, 1500 msm, 11-IV-86, Bianchetti et al. 429 (CORD). Est. São Paulo: Reserva Florestal da Bocaina, \pm 1600 msm, 5-V-1968, Sucre et al. 2907 (CORD). Est. Rio de Janeiro: Munic. Resende, Parque Nacional Itatiaia, 27-II-2006, Barboza et al. 1653 (CORD).

Área de estudio

Los materiales analizados proceden de seis Provincias Biogeográficas (Cabrera \& Willink, 1980): Paranaense (Argentina y Brasil): C. baccatum y C. flexuosum; de las Yungas (Argentina): $C$. baccatum; Mata Atlántica (Brasil): C. cornutum, $C$. friburgense, C. hunzikerianum, C. recurvatum y $C$. villosum; Pacífica (Colombia): C. geminifolium; de la Caatinga (Brasil): $C$. caatingae y $C$. longidentatum; Chaqueña (Argentina y Bolivia): $C$. cardenasii, $C$. chacoense y $C$. eximium. Las cuatro primeras regiones caracterizadas principalmente por su ambiente húmedo y las dos restantes por su ambiente seco.

Metodología: Se trabajó con material de herbario y material fresco fijado en FAA. Las hojas de los ejemplares de herbario fueron reconstituidas por imbibición en agua con una gota de detergente en estufa a $36^{\circ}-37^{\circ} \mathrm{C}$, durante $24-72 \mathrm{hs}$, previo a su fijación en FAA. El material fijado fue deshidratado en una serie ascendente de alcohol etílico, clarificado con xilol e incluido en parafina (Histowax ${ }^{\circledR}$ o Paramat ${ }^{\circledR}$ ). Los cortes transversales (12 a $15 \mu \mathrm{m}$ de espesor) fueron realizados con micrótomo rotatorio (Yidi, 7508), coloreados con hematoxilina activada, safranina y verde permanente (triple coloración) $\mathrm{y}$, finalmente, montados con bálsamo de Canadá (preparaciones permanentes) (Conn et al., 1960).

Para analizar la epidermis foliar en vista superficial se realizaron preparados temporarios utilizando la técnica de "peeling"; tanto la epidermis abaxial como la adaxial fueron coloreadas con safranina $y$ montadas en glicerina al 50\% (D’Ambrogio de Argüeso, 1986). En los casos donde la epidermis no se separó fácilmente del mesofilo, se utilizaron hojas diafanizadas en hipoclorito de sodio comercial 60\%. El material 
proveniente de herbario fue hidratado previamente en agua bajo el punto de ebullición, enjuagado con agua destilada y aclarado con hipoclorito de sodio comercial $50 \%$.

Para el análisis cuantitativo, se determinó el número de células epidérmicas propiamente dichas, de estomas y de tricomas por $\mathrm{mm}^{2}$ mediante un ocular reticulado. Para ello, de cada especie se tomaron tres individuos de distinta procedencia y de cada uno, cuatro hojas adultas de tamaño medio ubicadas en los $2 / 3$ de la longitud total de la planta. En cada preparado (pseudoréplica) se contaron al azar cinco campos. Los valores de las pseudoréplicas de cada especie fueron promediados y el resultado expresado como la densidad en $1 \mathrm{~mm}^{2}$ de lámina foliar.

El índice estomático se calculó según la siguiente fórmula (Stace, 1965):

I.E. $=$ (densidad de estomas/densidad de estomas + densidad de células epidérmicas). 100 .

Las variables epidérmicas: densidad de células epidérmicas propiamente dichas, de estomas, de tricomas eglandulares y glandulares e índice estomático se seleccionaron para el análisis estadístico. Todas las variables fueron analizadas con el programa InfoStat (Di Rienzo et al., 2011). Se calcularon las medias y desvíos estándares discriminando las caras foliares, para cada una de las especies. Además, se realizaron análisis de varianza paramétrica y no paramétrica (Kruskal Wallis), según se cumplieran los supuestos de normalidad y homogeneidad, con el objetivo de determinar si existen diferencias significativas entre las caras foliares de cada especie y entre las especies (Scheffé, 1959; Sokal \& Rohlf, 1981).

Todas las observaciones se efectuaron con microscopio óptico (Zeiss, Primo Star) y las fotomicrografías se tomaron con cámara digital (Nikon, Coolpix 5200). Las ilustraciones son originales y se realizaron con la ayuda de una cámara clara adosada al microscopio mencionado.

\section{Resultados}

\section{Epidermis en vista superficial}

Se observaron tres tipos de tricomas: Tipo I, eglandular simple: con 3 a 7 células, uniseriado, más o menos recto (Fig. 1 A-B) o curvo (Fig. 1 C-E) y con cutícula levemente a fuertemente rugosa (Fig. 1 A-E, Fig. 2 A-C); Tipo II, eglandular ramificado pluricelular, con 2 ó 3 ramas (Fig. 1 F, Fig. 2 D); Tipo III, glandular simple con pie unicelular y cabezuela pluricelular globosa o alargada y cutícula lisa (Fig. 1 G, Fig. 2 E-F). En general, en las especies analizadas se observan tricomas Tipo I y III, excepto C. caatingae que presenta sólo Tipo I, C. geminifolium sólo Tipo III y C. longidentatum sólo Tipo II y III (Tablas 1-3).

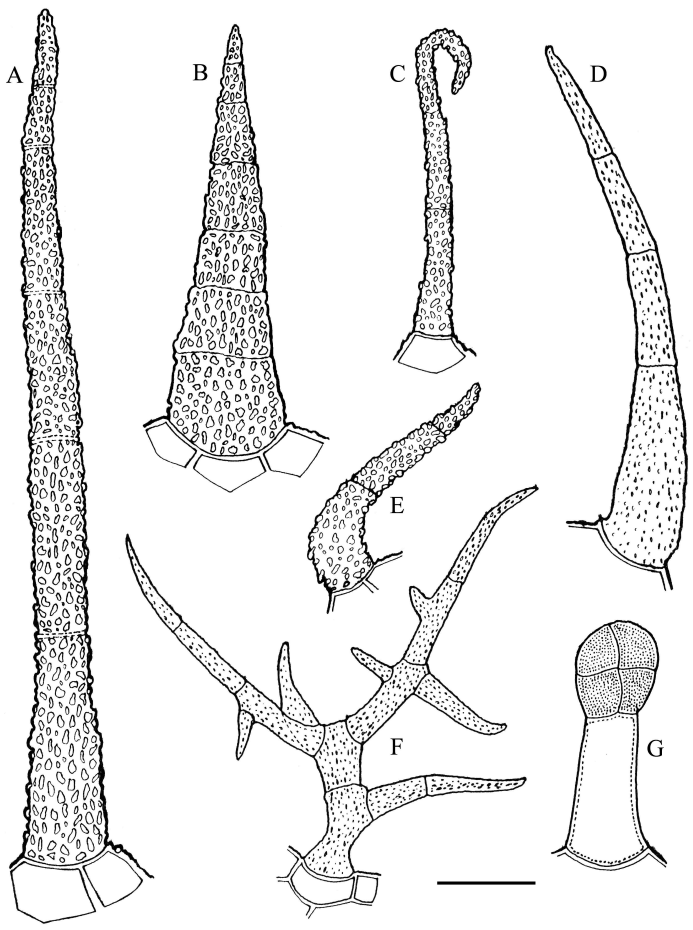

Fig. 1. Tipos de tricomas en Capsicum. A-B: Eglandular simple recto (Tipo I). C-E: Eglandular simple curvo (Tipo I). F: Eglandular ramificado (Tipo II). G: Glandular simple (Tipo III). Escalas: A-F: $100 \mu \mathrm{m}$, G: $50 \mu \mathrm{m}$.

Las células epidérmicas propiamente dichas de ambas caras foliares pueden ser de paredes anticlinales subrectas (Fig. 2 G, I), sinuosas (Fig. 2 $\mathrm{H}, \mathrm{J}-\mathrm{K}$ ) o ligeramente sinuosas (Fig. 2 L) y, en la mayoría de los casos, de forma irregular o alargada (Fig. 2 G-H, K), a veces isodiamétrica (Fig. 2 I, L) (Tablas 4-6).

Los estomas generalmente son escasos en la cara adaxial (Fig. 2 G-H) o excepcionalmente ausentes (Fig. 2 I), respecto a la abaxial (Fig. 2 J-L). Son de tipo anomocítico (Fig. 2 J), anisocítico (Fig. 2 L) o hemiparacítico (Fig. 2 K) (Tablas 1-3).

\section{Estructura interna de la lámina}

En sección transversal se observa epidermis unistrata, cutícula lisa o levemente estriada, delgada 
M. P. Palchetti et al. - Anatomía foliar en Capsicum
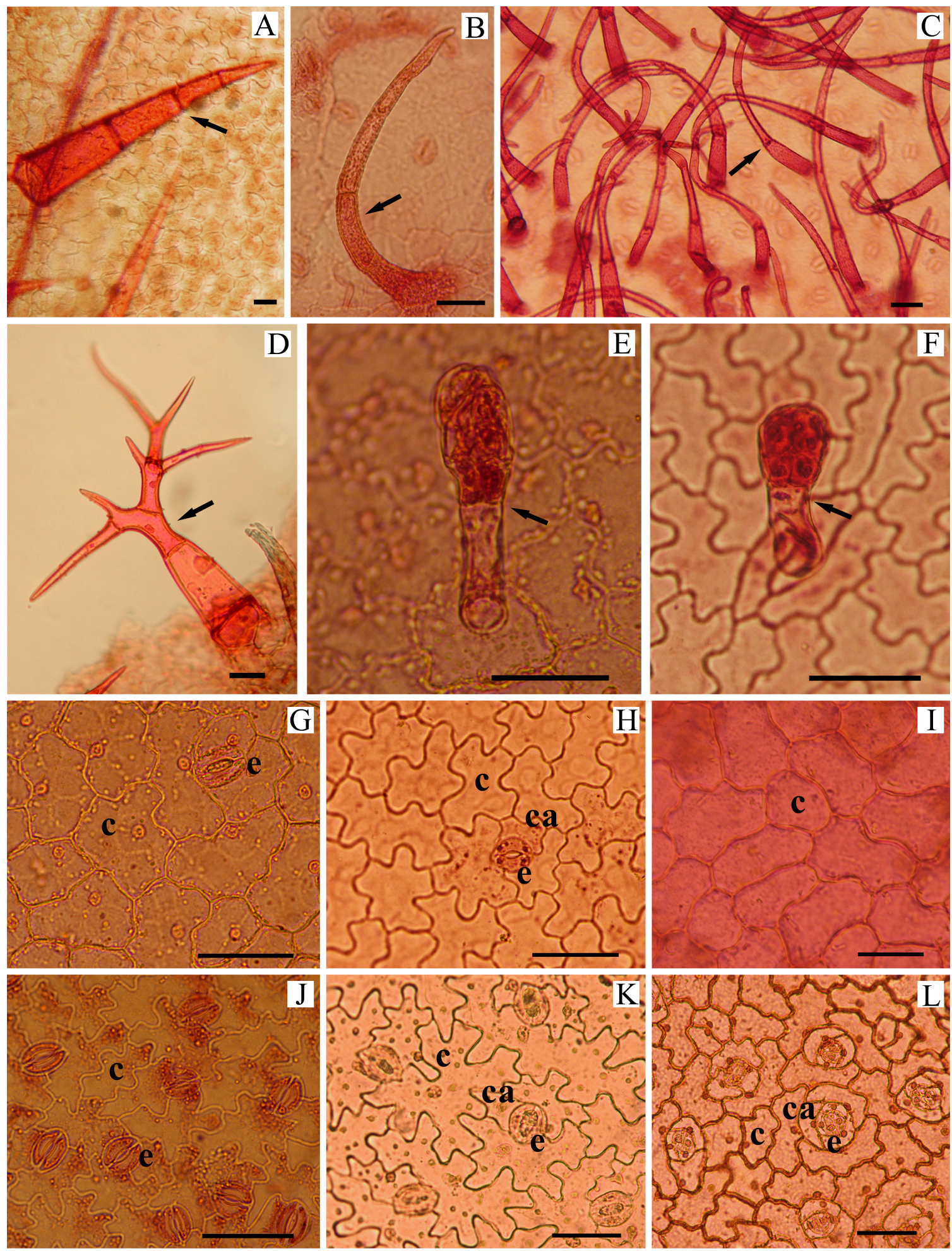

Fig. 2. Epidermis foliar en vista superficial. A-C: Tricomas eglandulares simples. D: Tricoma eglandular ramificado, Capsicum longidentatum. E-F: Tricomas glandulares simples. G-I: Cara adaxial. J-L: Cara abaxial. A, K: C. cornutum. B, H, F: C. chacoense. C: C. caatingae. E, G, J: C. baccatum. I, L: C. geminifolium. Referencias, c: célula epidérmica propiamente dicha, ca: célula anexa, e: estoma. Escala: $50 \mu \mathrm{m}$. 
Bol. Soc. Argent. Bot. 49 (3) 2014
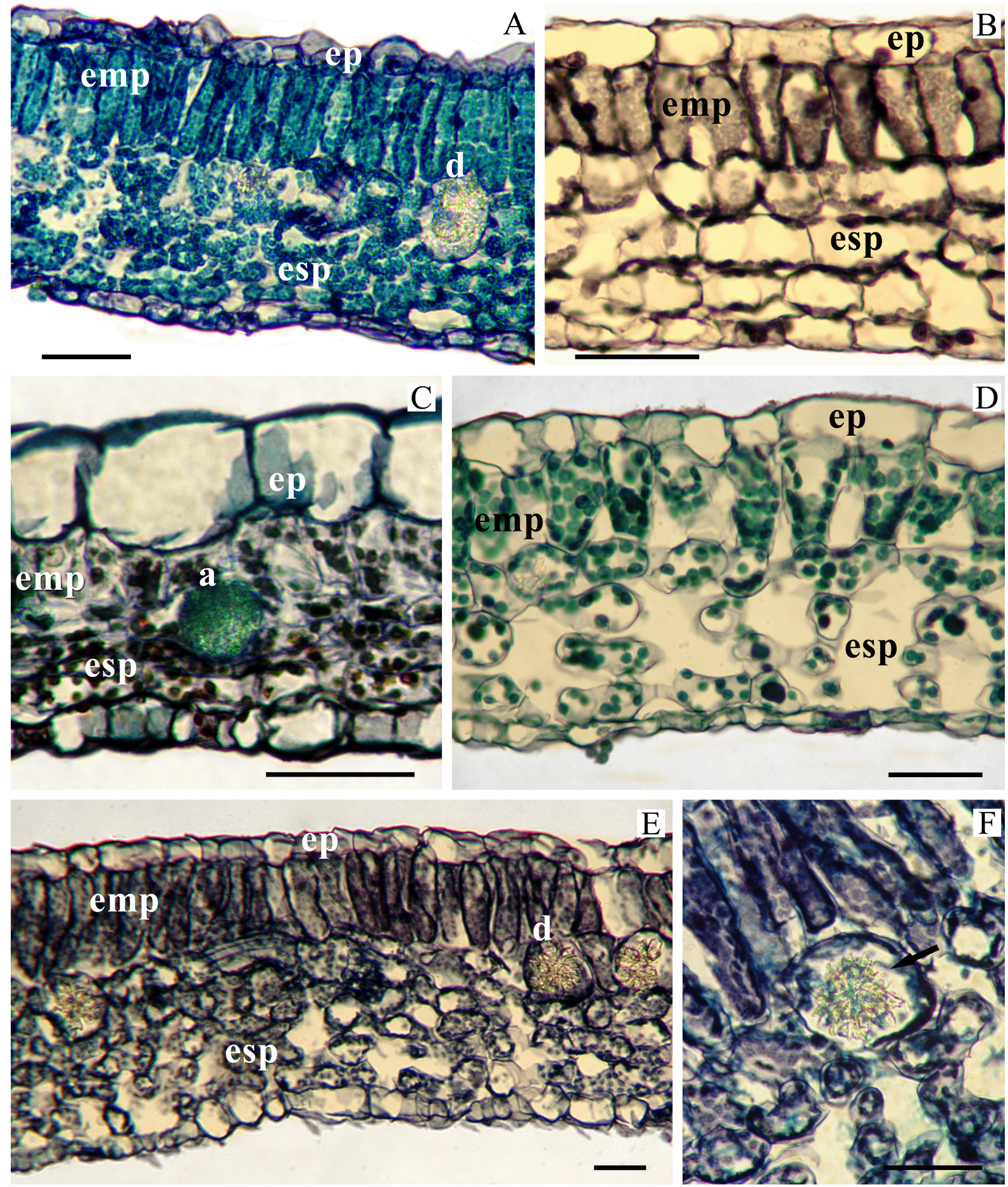

Fig. 3. Corte transversal de la hoja, lámina. A: Capsicum baccatum. B: C. flexuosum. C: C. geminifolium. D: C. cornutum. E-F: C. friburgense, detalle de drusa (flecha). Referencias, a: areniscas, d: drusa, emp: parénquima en empalizada, ep: epidermis, esp: parénquima esponjoso. Escala: $50 \mu \mathrm{m}$. 
Tabla 1. Epidermis en vista superficial de las hojas de las especies de Capsicum que habitan en las Provincias Biogeográficas Paranaense, de las Yungas y Pacífica (ambiente húmedo). Abreviaturas, aniso: anisocítico, anomo: anomocítico, cep: célula epidérmica propiamente dicha, hemip: hemiparacítico.

\begin{tabular}{|c|c|c|c|c|}
\hline & $\begin{array}{l}\text { Provincias } \\
\text { Biogeográficas }\end{array}$ & Paranaense y De las Yungas & Paranaense & Pacífica \\
\hline & TAXONES & \multirow[t]{2}{*}{ C. baccatum } & \multirow[t]{2}{*}{ C. flexuosum } & \multirow[t]{2}{*}{ C. geminifolium } \\
\hline & CARACTERES & & & \\
\hline \multirow{4}{*}{$\begin{array}{l}\frac{\pi}{\frac{\pi}{x}} \\
\frac{\pi}{0} \\
\frac{\pi}{<}\end{array}$} & Estomas & Hemip & Anomo y hemip & Ausentes \\
\hline & Forma cep & Irregular o alargada & Alargada & Alargada o isodiamétrica \\
\hline & Paredes & Subrectas & Ligeramente sinuosas & Subrectas \\
\hline & Tricomas & I y III & I y III & III \\
\hline \multirow{4}{*}{ 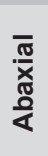 } & Estomas & Anomo y hemip & Anomo y hemip & Aniso \\
\hline & Forma cep & Alargada o casi isodiamétrica & Alargada o irregular & Alargada o isodiamétrica \\
\hline & Paredes & Sinuosas & Sinuosas & Ligeramente sinuosas \\
\hline & Tricomas & I y |II & I y III & III \\
\hline
\end{tabular}

Tabla 2. Epidermis en vista superficial de las hojas de las especies de Capsicum que habitan en la Mata Atlántica (ambiente húmedo). Abreviaturas, aniso: anisocítico, anomo: anomocítico, cep: célula epidérmica propiamente dicha, hemip: hemiparacítico.

\begin{tabular}{|c|c|c|c|c|c|c|}
\hline & $\begin{array}{l}\text { Provincia } \\
\text { Biogeográfica }\end{array}$ & Mata Atlántica & Mata Atlántica & Mata Atlántica & Mata Atlántica & $\begin{array}{c}\text { Mata } \\
\text { Atlántica }\end{array}$ \\
\hline & $\begin{array}{l}\text { TAXONES } \\
\text { CARACTERES }\end{array}$ & C. cornutum & C. friburgense & C. hunzikerianum & C. recurvatum & C. villosum \\
\hline \multirow{4}{*}{$\begin{array}{l}\bar{\pi} \\
\frac{\pi}{x} \\
\frac{\pi}{0} \\
\end{array}$} & Estomas & Ausentes & Hemip & Hemip & Aniso, a veces anomo & Aniso \\
\hline & Forma cep & $\begin{array}{l}\text { Irregular o } \\
\text { alargada }\end{array}$ & Irregular & Irregular & $\begin{array}{c}\text { Irregular o } \\
\text { isodiamétrica }\end{array}$ & $\begin{array}{l}\text { Irregular o } \\
\text { alargada }\end{array}$ \\
\hline & Paredes & Sinuosas & Sinuosas & Sinuosas & Ligeramente sinuosas & Muy sinuosas \\
\hline & Tricomas & I y ||| & I y |II & I y |II & I y III & I y III \\
\hline \multirow{4}{*}{$\begin{array}{l}\bar{\pi} \\
\bar{x} \\
\frac{\pi}{\alpha}\end{array}$} & Estomas & Hemip & Hemip & Anomo y hemip & Aniso, a veces anomo & $\begin{array}{l}\text { Aniso y } \\
\text { anomo }\end{array}$ \\
\hline & Forma cep & Irregular & Irregular & Irregular & Irregular & Irregular \\
\hline & Paredes & Muy sinuosas & Sinuosas & Muy sinuosas & Sinuosas & Muy sinuosas \\
\hline & Tricomas & I y III & I y |II & I y III & I y III & I y III \\
\hline
\end{tabular}

o gruesa según las especies. El mesofilo posee una estructura dorsiventral con un solo estrato de parénquima en empalizada y dos a seis capas de parénquima esponjoso con espacios intercelulares que varían de pequeños a grandes (Fig. 3 A-E, 4 BE). En el parénquima en empalizada y en las células epidérmicas de $C$. caatingae se ha observado citoplasma con contenido denso, debido probablemente a la presencia de taninos (Fig. 4 E, 5 F). En la vena media, el haz vascular es bicolateral en todas las especies y está rodeado por células parenquimáticas, excepto en $C$. geminifolium que tiene fibras acompañando al floema (Fig. 5 C) y $C$. chacoense que posee una capa de colénquima. En la mayoría de las especies, se observa también colénquima subepidérmico distribuido en 1-4 capas en ambas superficies de la vena media. Los cristales son bastante frecuentes en casi todas las especies, pudiendo ser areniscas y/o drusas; C. cardenasii presenta, además, cristales solitarios romboédricos en células del mesofilo (Tablas 4-6).

\section{Estructura interna del peciolo}

Posee epidermis unistrata. El haz vascular es bicolateral y tiene forma de arco (Fig. 6 B-F), salvo C. geminifolium donde es en U (Fig. 6 A). Sólo $C$. friburgense carece de colénquima mientras que en las restantes especies, este tejido se encuentra distribuido en una a seis capas subepidérmicas. Todas las especies presentan areniscas cristalinas, salvo $C$. eximium que además presenta drusas y $C$. flexuosum que no posee ningún tipo de cristales (Tablas 4-6).

Particularidades anatómicas por especie

Las particularidades anatómicas de la lámina y pecíolo de cada especie, agrupadas según la 
Tabla 3. Epidermis en vista superficial de las hojas de las especies de Capsicum que habitan en las Provincias Biogeográficas Chaqueña y de la Caatinga (ambiente seco). Abreviaturas, aniso: anisocítico, anomo: anomocítico, cep: célula epidérmica propiamente dicha, hemip: hemiparacítico.

\begin{tabular}{|c|c|c|c|c|c|c|}
\hline & $\begin{array}{l}\text { Provincias } \\
\text { Biogeográficas }\end{array}$ & Chaqueña & Chaqueña & Chaqueña & De la Caatinga & De la Caatinga \\
\hline & TAXONES & \multirow[t]{2}{*}{ C. cardenasii } & \multirow[t]{2}{*}{ C. chacoense } & \multirow[t]{2}{*}{ C. eximium } & \multirow[t]{2}{*}{ C. caatingae } & \multirow[t]{2}{*}{ C. longidentatum } \\
\hline & CARACTERES & & & & & \\
\hline \multirow{4}{*}{$\begin{array}{l}\frac{\pi}{x} \\
\frac{\pi}{0} \\
\frac{0}{4}\end{array}$} & Estomas & Aniso & $\begin{array}{l}\text { Hemip, a veces } \\
\text { anomo }\end{array}$ & $\begin{array}{c}\text { Anomo, a veces } \\
\text { hemip }\end{array}$ & Ausentes & Hemip \\
\hline & Forma cep & $\begin{array}{c}\text { Irregular, a } \\
\text { veces alargada }\end{array}$ & Irregular o alargada & $\begin{array}{l}\text { Irregular o } \\
\text { alargada }\end{array}$ & Irregular & Irregular \\
\hline & Paredes & $\begin{array}{l}\text { Ligeramente } \\
\text { sinuosas }\end{array}$ & Sinuosas & Sinuosas & Sinuosas & Muy sinuosas \\
\hline & Tricomas & I y III & I y |II & I y |II & 1 & II y III \\
\hline \multirow{4}{*}{$\begin{array}{l}\overline{\frac{\pi}{x}} \\
\frac{X}{\pi} \\
\frac{0}{\alpha}\end{array}$} & Estomas & Aniso & $\begin{array}{c}\text { Hemip, a veces } \\
\text { anomo }\end{array}$ & $\begin{array}{c}\text { Anomo, a veces } \\
\text { hemip }\end{array}$ & Anomo y hemip & Anomo \\
\hline & Forma cep & $\begin{array}{c}\text { Irregular, a } \\
\text { veces alargada }\end{array}$ & Irregular & Irregular & Irregular & Irregular \\
\hline & Paredes & Sinuosas & Sinuosas & Sinuosas & Sinuosas & Muy sinuosas \\
\hline & Tricomas & I y |II & I y |II & I y III & I & II y III \\
\hline
\end{tabular}

Provincia Biogeográfica a la que pertenecen, se detallan en las Tablas 1 a 6.

En C. cardenasii (Provincia Biogeográfica Chaqueña) no se pudieron observar, en su totalidad, detalles de la estructura interna de la hoja. Sin embargo, se pudo apreciar que posee abundantes areniscas cristalinas alrededor del haz vascular, tanto de la lámina como del pecíolo y abundantes drusas y cristales romboédricos en el mesofilo (Fig. 4 F-G, 6 F).

\section{Análisis cuantitativo}

Los resultados del análisis de la varianza muestran que la densidad de estomas, de células epidérmicas y el índice estomático son significativamente diferentes entre las caras foliares de la mayoría de las especies estudiadas, siendo en todos los casos mayor en la abaxial. En $C$. baccatum, C. caatingae, C. cardenasii y C. eximium no se encontraron diferencias significativas en la densidad de las células epidérmicas propiamente dichas entre caras foliares (Tabla 7).

La densidad de tricomas eglandulares no difiere significativamente entre las caras de las epidermis foliares, excepto $C$. eximium, donde es menor en la abaxial y en C. hunzikerianum, mayor en la abaxial. Por último, la densidad de tricomas glandulares sólo es significativamente diferente entre las epidermis de $C$. baccatum, $C$. eximium, $C$. friburgense, $C$. geminifolium, $C$. longidentatum, $C$. recurvatum y $C$. villosum, siendo mayor en la abaxial (Tabla 7).
Luego se compararon la densidad de estomas y tricomas glandulares y eglandulares de la epidermis abaxial y adaxial entre las especies en estudio mediante análisis de la varianza no paramétricos $(\mathrm{p}<0,05)$, observándose diferencias significativas en estas variables (Tabla 8-10).

Los resultados de la densidad de estomas de la epidermis abaxial mostraron que $C$. longidentatum es una de las especies con valores más bajos y $C$. recurvatum la de mayor valor. En cambio, en la epidermis adaxial se diferencian significativamente C. geminifolium, C. cornutum y C. caatingae con la menor densidad estomática y $C$. chacoense, $C$. recurvatum y $C$. cardenasii por poseer los valores más altos (Tabla 8).

En cuanto a densidad de tricomas eglandulares (Tipo I o II según la especie) en la epidermis abaxial, $C$. caatingae, $C$. longidentatum, $C$. cornutum y $C$. hunzikerianum tienen valores significativamente mayores al resto de las especies. En la epidermis adaxial, C. geminifolium es la especie que posee el menor valor y $C$. longidentatum el mayor valor (Tabla 9).

Finalmente, la densidad de tricomas glandulares en la epidermis abaxial presenta un grupo bien diferenciado compuesto por $C$. caatingae, $C$. cornutum, $C$. flexuosum y $C$. chacoense, que presentan los menores valores, después le siguen el resto de las especies finalizando en $C$. eximium con el máximo valor. En la epidermis adaxial, $C$. eximium, $C$. hunzikerianum y $C$. cardenasii tienen los más altos valores (Tabla 10). 
M. P. Palchetti et al. - Anatomía foliar en Capsicum
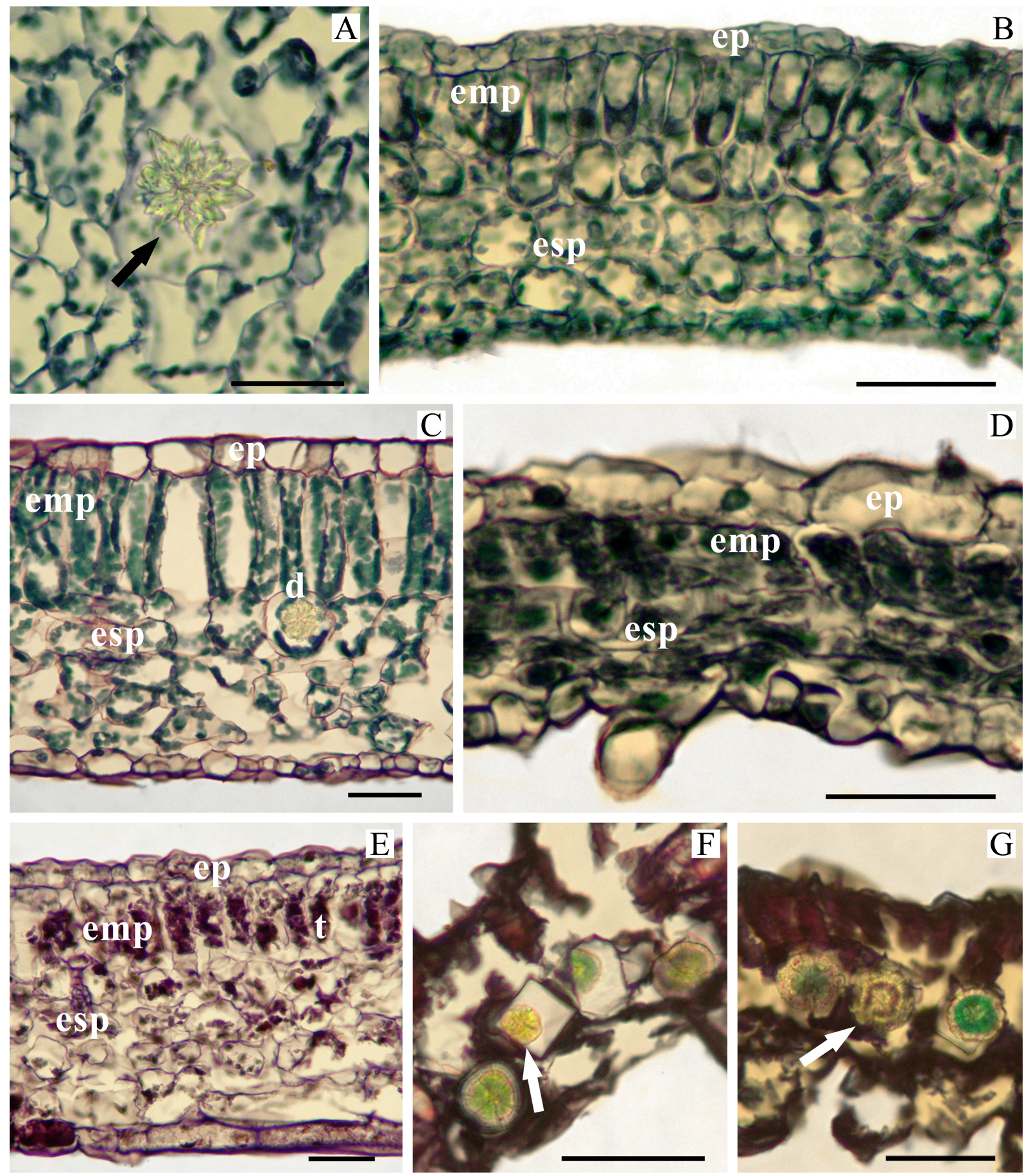

Fig. 4. Corte transversal de la hoja, lámina. A: Capsicum hunzikerianum, detalle de drusa (flecha). B: C. recurvatum. C: C. chacoense. D: C. eximium. E: C. caatingae. F: $C$. cardenasii, detalle de cristales romboédricos (flecha). G: C. cardenasii, detalle de drusa (flecha). Referencias, d: drusa, emp: parénquima en empalizada, ep: epidermis, esp: parénquima esponjoso, t: probables taninos. Escala: $40 \mu \mathrm{m}$. 
Bol. Soc. Argent. Bot. 49 (3) 2014

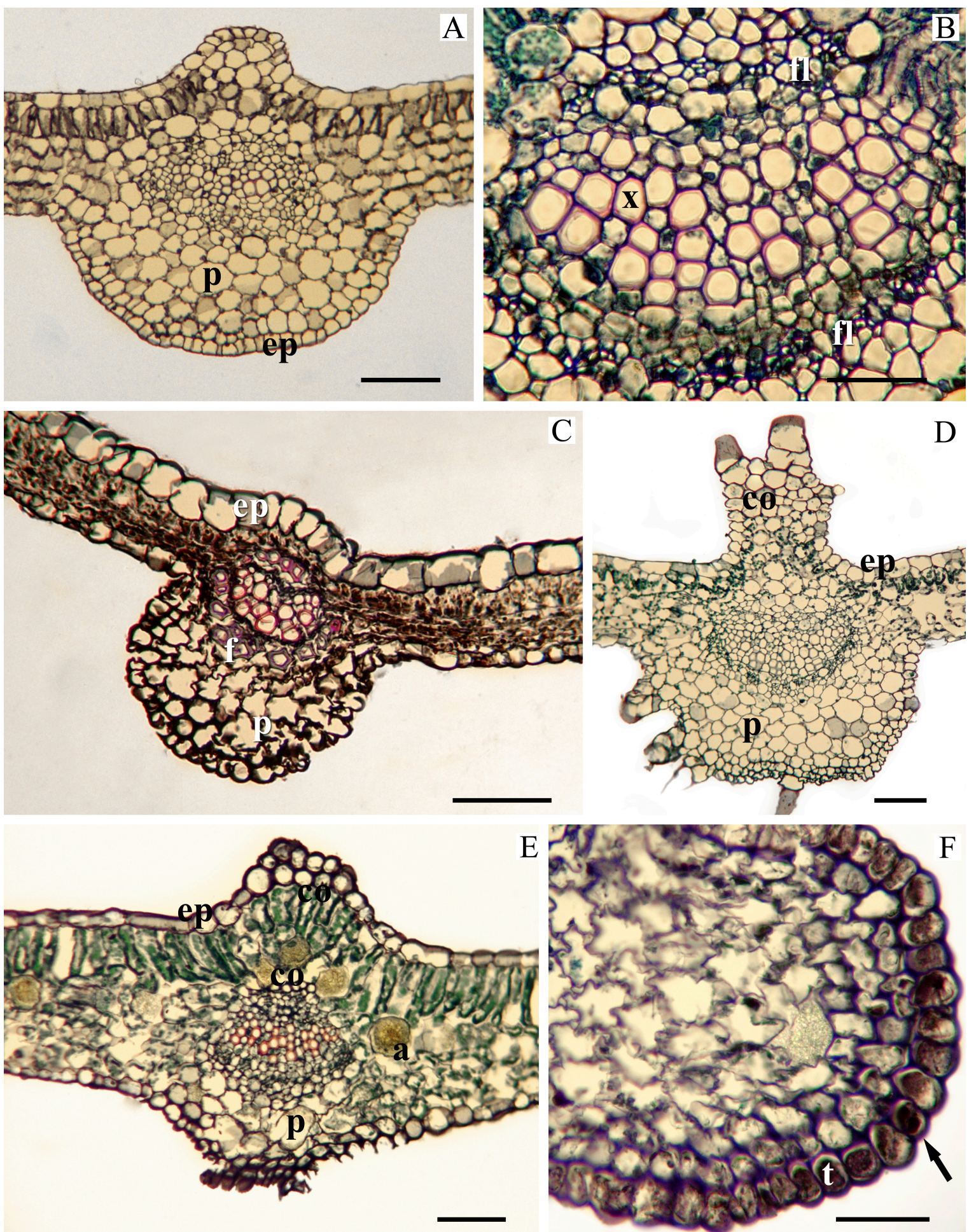

Fig. 5. Corte transversal de la hoja, vena media. A: Capsicum flexuosum. B: C. baccatum, detalle de haz vascular. C: C. geminifolium. D: C. cornutum. E: C. chacoense. F: C. caatingae, detalle de parte de vena media. Referencias, a: areniscas, co: colénquima, ep: epidermis, f: fibras, fl: floema, p: parénquima, t: probables taninos, x: xilema. Escalas: A, C-E: $100 \mu \mathrm{m}$; B, F: $50 \mu \mathrm{m}$. 

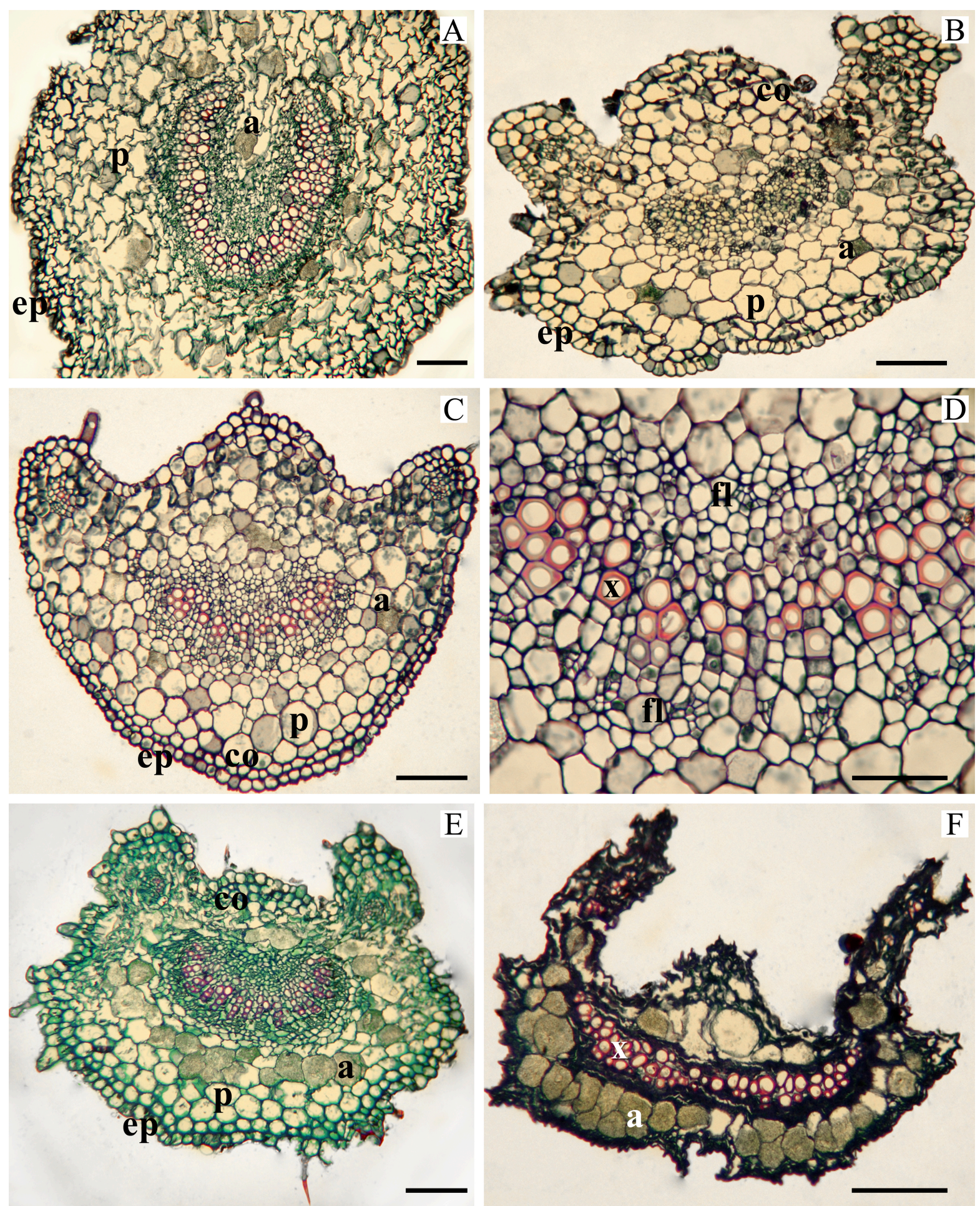

Fig. 6. Corte transversal de pecíolo. A: Capsicum geminifolium. B: C. hunzikerianum. C-D: C. chacoense, detalle de haz vascular. E. C. Iongidentatum. F. C. cardenasii. Referencias, a: areniscas; co: colénquima, ep: epidermis, fl: floema, p: parénquima, $\mathbf{x}$ : xilema. Escalas: A-C, E-F: $100 \mu \mathrm{m}$; D: $50 \mu \mathrm{m}$. 
Tabla 4. Anatomía de lámina y pecíolo de las especies de Capsicum que habitan en las Provincias

Biogeográficas Paranaense, de las Yungas y Pacífica (ambiente húmedo). Abreviaturas, abax: abaxial, adax: adaxial, co: colénquima, emp: parénquima en empalizada, esp: parénquima esponjoso, pa: parénquima, subepi: subepidérmico, >: mayores en.

\begin{tabular}{|c|c|c|c|c|}
\hline & $\begin{array}{l}\text { Provincias } \\
\text { Biogeográficas }\end{array}$ & Paranaense y De las Yungas & Paranaense & Pacífica \\
\hline & TAXONES & C. baccatum (Fig. 3 A, 5 B) & C. flexuosum (Fig. 3 B, 5 A) & $\begin{array}{l}\text { C. geminifolium (Fig. } 3 \mathrm{C} \text {, } \\
5 \mathrm{C}, 6 \mathrm{~A})\end{array}$ \\
\hline & CARACTERES & & & \\
\hline \multirow{9}{*}{$\underset{\Sigma}{\stackrel{\Xi}{Z}}$} & Espesor & $150 \mathrm{~mm}$ & $110 \mathrm{~mm}$ & $120 \mathrm{~mm}$ \\
\hline & Cél. epidérmicas & Rectangulares & Rectangulares & $\begin{array}{c} \pm \text { rectangulares, }>\text { cara } \\
\text { adaxial }\end{array}$ \\
\hline & Cutícula & Gruesa & Delgada & Delgada \\
\hline & Parénquima esponjoso & $\begin{array}{c}\text { 5-6 capas, medianos espacios } \\
\text { intercelulares }\end{array}$ & $\begin{array}{c}3 \text { capas, medianos } \\
\text { espacios intercelulares }\end{array}$ & $\begin{array}{c}4 \text { capas, pequeños } \\
\text { espacios intercelulares }\end{array}$ \\
\hline & Relación emp/esp & $3 / 4$ & 1 & $1 / 2$ \\
\hline & Cristales & Areniscas y drusas & Ausentes & Areniscas \\
\hline & \multirow{3}{*}{ Vena media } & Convexa en ambas caras. & Convexa en abax. & Convexa en abax. \\
\hline & & Emp continuo. & Emp discontinuo. & Emp continuo. \\
\hline & & $\begin{array}{c}1 \text { capa de co subepi en ambas } \\
\text { caras }\end{array}$ & Sin co & Sin co \\
\hline \multirow{4}{*}{$\frac{0}{0}$} & Cutícula & Ligeramente gruesa y estriada & Delgada y lisa & Delgada y lisa \\
\hline & Cristales & Areniscas & Ausentes & Areniscas \\
\hline & \multirow{2}{*}{ Tejidos fundamentales } & $2-3$ capas de co subepi & 2-3 capas de co subepi & 2-3 capas de co subepi \\
\hline & & Sin fibras & Sin fibras & Fibras en floema interno \\
\hline
\end{tabular}

\section{DISCUSIÓN Y CONCLUSIONES}

Las especies de Capsicum estudiadas presentan una gran similitud en la estructura interna de las hojas. Se observaron estomas anisocíticos, anomocíticos y hemiparacíticos. La presencia de varios tipos de estomas en una misma hoja es un carácter común en Solanaceae (Bessis \& Guyot, 1979; Karatela \& Gill, 1986; Liscovsky \& Cosa, 2005), diferenciándose en ambas caras foliares o sólo en la abaxial (Metcalfe \& Chalk, 1983). En nuestro estudio, las hojas son hipostomáticas en $C$. caatingae, $C$. cornutum y C. geminifolium; anfistomáticas, con escasos (C. baccatum, $C$. eximium, $C$. flexuosum, $C$. friburgense, $C$. hunzikerianum, C. longidentatum y C. villosum) o con frecuentes estomas en la epidermis adaxial $(C$. cardenasii, $C$. chacoense y $C$. recurvatum). La presencia de abundantes estomas en ambas caras de las hojas es un carácter común en xerófitos ya que permite el rápido intercambio de gases durante el poco tiempo que están abiertos, evitando así la pérdida de agua por transpiración (Fahn, 1990; Fahn \& Cutler, 1992). Sin embargo, otros autores (Thakur, 1990; Toral et al., 2010) señalaron que la disminución en la densidad incrementa la resistencia estomática y evita el exceso de transpiración. La densidad de estomas en todas las especies aquí estudiadas es comparativamente mucho más alta en la epidermis abaxial que en la adaxial y las especies con mayores valores habitan en ambientes mesofíticos, excepto $C$. eximium que habita en ambiente relativamente seco. Resultados similares fueron reportados por Wood (1934) en plantas de bosques esclerófilos del sur de Australia donde la densidad estomática es baja y no informó variación significativa con respecto a plantas de ambientes mesofíticos. Dichas particularidades al parecer estarían más relacionadas con caracteres genéricos y de familia que a condiciones ambientales.

Las paredes de las células epidérmicas de Capsicum variaron desde subrectas a muy sinuosas, presentando ambas características especies de uno y otro ambiente; por otra parte, se sabe que las ondulaciones de las paredes epidérmicas es un carácter variable, por lo que su uso en taxonomía debe hacerse con precaución (Stace, 1965). En este trabajo, tales ondulaciones no resultaron diagnósticas para distinguir las especies de Capsicum ni para caracterizar especies en un determinado ambiente.

En Solanáceas es común la presencia de abundantes y variados tipos de tricomas (Roe, 1972; Seithe, 1979; Metcalfe \& Chalk, 1983; Mentz et al., 


\section{V. Palchetti et al. - Anatomía foliar en Capsicum}

Tabla 5. Anatomía de lámina y pecíolo de las especies de Capsicum que habitan en la Mata Atlántica

(ambiente húmedo). Referencias, abax: abaxial, adax: adaxial, cél.: células, co: colénquima, emp: parénquima en empalizada, esp: parénquima esponjoso, pa: parénquima, subepi: subepidérmico, >: mayores en.

\begin{tabular}{|c|c|c|c|c|c|c|}
\hline & $\begin{array}{l}\text { Provincia } \\
\text { Biogeográfica }\end{array}$ & Mata Atlántica & Mata Atlántica & Mata Atlántica & Mata Atlántica & Mata Atlántica \\
\hline & TAXONES & $\begin{array}{l}\text { C. cornutum } \\
\text { (Fig. } 3 \text { D, } 5 \text { D) }\end{array}$ & $\begin{array}{l}\text { C.friburgense } \\
\text { (Fig. } 3 \mathrm{E}-\mathrm{F} \text { ) }\end{array}$ & $\begin{array}{l}\text { C. hunzikerianum } \\
\text { (Fig. } 4 \text { A, } 6 \text { B) }\end{array}$ & $\begin{array}{l}\text { C.recurvatum } \\
\text { (Fig. } 4 \text { B) }\end{array}$ & C. villosum \\
\hline & \multicolumn{6}{|l|}{ CARACTERES } \\
\hline \multirow{9}{*}{$\frac{\mathbb{Z}}{\sum}$} & Espesor & $240 \mathrm{~mm}$ & 300 mm & $170 \mathrm{~mm}$ & $90 \mathrm{~mm}$ & 115 mm \\
\hline & Cél. epidérmicas & $\begin{array}{l}\text { Rectangulares a } \\
\text { isodiamétricas }\end{array}$ & Rectangulares & $\begin{array}{l}\text { Rectangulares, } \\
>\text { cara adaxial }\end{array}$ & Rectangulares & $\begin{array}{c}\text { Rectangulares }>\text { cara } \\
\text { adaxial }\end{array}$ \\
\hline & Cutícula & Delgada & $\begin{array}{l}\text { Ligeramente } \\
\text { gruesa }\end{array}$ & Delgada & Delgada & Ligeramente gruesa \\
\hline & $\begin{array}{l}\text { Parénquima } \\
\text { esponjoso }\end{array}$ & $\begin{array}{c}4 \text { capas, } \\
\text { grandes } \\
\text { espacios } \\
\text { intercelulares }\end{array}$ & $\begin{array}{l}\text { 4-5 capas, } \\
\text { medianos } \\
\text { espacios } \\
\text { intercelulares }\end{array}$ & $\begin{array}{c}3 \text { capas, medianos } \\
\text { espacios } \\
\text { intercelulares }\end{array}$ & $\begin{array}{c}3 \text { capas, } \\
\text { pequeños } \\
\text { espacios } \\
\text { intercelulares }\end{array}$ & $\begin{array}{c}\text { 3-4 capas, grandes } \\
\text { espacios } \\
\text { intercelulares }\end{array}$ \\
\hline & $\begin{array}{l}\text { Relación } \\
\text { emp/esp }\end{array}$ & $3 / 5$ & $1 / 2$ & 1 & $1 / 2$ & $1 / 2$ \\
\hline & Cristales & Drusas & $\begin{array}{c}\text { Areniscas y } \\
\text { drusas }\end{array}$ & $\begin{array}{c}\text { Areniscas y } \\
\text { drusas }\end{array}$ & Ausentes & Drusas \\
\hline & \multirow{3}{*}{ Vena media } & $\begin{array}{l}\text { Convexa en } \\
\text { ambas caras. }\end{array}$ & $\begin{array}{l}\text { Convexa en } \\
\text { ambas caras. }\end{array}$ & Convexa en abax. & $\begin{array}{l}\text { Convexa en } \\
\text { ambas caras. }\end{array}$ & Convexa en abax. \\
\hline & & Emp continuo. & $\begin{array}{c}\text { Emp } \\
\text { discontinuo. }\end{array}$ & Emp continuo. & Emp discontinuo. & Emp continuo. \\
\hline & & $\begin{array}{c}\text { 3-4 capas de co } \\
\text { subepi en adax } \\
\text { y } 1 \text { en abax }\end{array}$ & $\begin{array}{c}4 \text { capas de co } \\
\text { subepi en adax } \\
\text { y } 2 \text { en abax }\end{array}$ & $\begin{array}{c}2 \text { capas de co } \\
\text { subepi en adax y } 1 \\
\text { en abax }\end{array}$ & $\begin{array}{l}2 \text { capas de co } \\
\text { subepi en abax }\end{array}$ & $\begin{array}{c}1 \text { capas de co subepi } \\
\text { en ambas caras }\end{array}$ \\
\hline \multirow{4}{*}{$\frac{0}{0}$} & Cutícula & Delgada y lisa & Delgada y lisa & Delgada y lisa & Delgada y lisa & Delgada y lisa \\
\hline & Cristales & Areniscas & Areniscas & Areniscas & Areniscas & Areniscas \\
\hline & \multirow[t]{2}{*}{$\begin{array}{l}\text { Tejidos } \\
\text { fundamentales }\end{array}$} & $\begin{array}{l}\text { Hasta } 6 \text { capas } \\
\text { de co subepi en } \\
\text { ambas caras }\end{array}$ & Sin co & $\begin{array}{c}3 \text { capas de co } \\
\text { subepi en adax y } 1 \\
\text { en abax }\end{array}$ & $\begin{array}{c}\text { 2-4 capas de co } \\
\text { subepi en ambas } \\
\text { caras }\end{array}$ & $\begin{array}{l}3 \text { capas de co subepi } \\
\text { en adax y } 1 \text { en abax }\end{array}$ \\
\hline & & Sin fibras & Sin fibras & Sin fibras & Sin fibras & Sin fibras \\
\hline
\end{tabular}

2000; Hunziker, 2001; Rigonatto et al., 2005). Nuestras observaciones señalan tres tipos de tricomas; eglandulares simples (Tipo I), eglandulares ramificados (Tipo II) y glandulares (Tipo III). La presencia de tricomas influye en la economía del agua ya sea directa o indirectamente (Fahn \& Cutler, 1992) y contribuye directamente a disminuir el movimiento del aire en la superficie que rodea la epidermis, permitiendo así concentrar el vapor de agua y evitar la excesiva transpiración. Este proceso se realiza, indirectamente, a través de la regulación de la temperatura mediante la reducción de la absorción de energía radiante o por reflexión de la luz. Esto concuerda con lo observado en las cuatro especies de ambientes secos: $C$. longidentatum y $C$. caatingae que habitan en ambientes de extrema sequía (de la Caatinga brasileña) (Barboza et al., 2011) y muestran abundantes tricomas eglandulares, y $C$. cardenasii y $C$. eximium, de ambiente chaqueño, también con abundantes tricomas, aunque en este caso glandulares y comparativamente muy pequeños. Sin embargo, también encontramos especies de ambientes húmedos con numerosos tricomas $\mathrm{y}$ otras de ambientes secos con escasos tricomas. Esta última situación fue señalada por Cutler (1987) para muchas xerófitas donde los tricomas son raros.

En relación a la anatomía de la lámina, las características generales como la estructura dorsiventral, la presencia de floema interno y de cristales, coinciden con las descriptas para la familia (Metcalfe \& Chalk, 1957; Cosa, 1991, 1993; Hunziker, 2001). Respecto a esto último, todas las especies, excepto C. flexuosum, presentaron cristales 
Bol. Soc. Argent. Bot. 49 (3) 2014

Tabla 6. Anatomía de lámina y pecíolo en corte transversal de las especies de Capsicum que habitan en las
Provincias Biogeográficas Chaqueña y de la Caatinga (ambiente seco). Abreviaturas, abax: abaxial, adax:
adaxial, cél.: células, co: colénquima, emp: parénquima en empalizada, esp: parénquima esponjoso, pa:
parénquima, subepi: subepidérmico, >: mayores en.

\begin{tabular}{|c|c|c|c|c|c|}
\hline & $\begin{array}{l}\text { Provincias } \\
\text { Biogeográficas }\end{array}$ & Chaqueña & Chaqueña & De la Caatinga & De la Caatinga \\
\hline & TAXONES & $\begin{array}{c}\text { C. chacoense (Fig. } 4 \mathrm{C} \text {, } \\
5 \mathrm{E}, 6 \mathrm{C}-\mathrm{D})\end{array}$ & C. eximium (Fig. 4 D) & C. caatingae (Fig. 4 E) & $\begin{array}{l}\text { C. longidentatum } \\
\text { (Fig. } 6 \mathrm{E})\end{array}$ \\
\hline \multirow{10}{*}{$\frac{\nwarrow}{\sum}$} & \multicolumn{5}{|l|}{ CARACTERES } \\
\hline & Espesor & $175 \mathrm{~mm}$ & $60 \mathrm{~mm}$ & $170 \mathrm{~mm}$ & $160 \mathrm{~mm}$ \\
\hline & Cél. epidérmicas & $\begin{array}{c}\text { Rectangulares, > cara } \\
\text { adaxial }\end{array}$ & Rectangulares & Rectangulares & Rectangulares \\
\hline & Cutícula & $\begin{array}{l}\text { Delgada en adax y } \\
\text { ligeramente gruesa en } \\
\text { abax }\end{array}$ & Gruesa & Ligeramente gruesa & Gruesa \\
\hline & $\begin{array}{l}\text { Parénquima } \\
\text { esponjoso }\end{array}$ & $\begin{array}{l}5 \text { capas, grandes } \\
\text { espacios intercelulares }\end{array}$ & $\begin{array}{l}2 \text { capas, pequeños } \\
\text { espacios intercelulares }\end{array}$ & $\begin{array}{l}4 \text { capas, medianos } \\
\text { espacios intercelulares }\end{array}$ & $\begin{array}{l}\text { 3-4 capas, } \\
\text { grandes espacios } \\
\text { intercelulares }\end{array}$ \\
\hline & \multirow{4}{*}{$\begin{array}{l}\text { Relación emp/esp } \\
\text { Cristales }\end{array}$} & $3 / 4$ & 1 & $3 / 5$ & 1 \\
\hline & & Areniscas y drusas & Areniscas y drusas & Areniscas & Areniscas \\
\hline & & $\begin{array}{c}\text { Convexa en ambas } \\
\text { caras. }\end{array}$ & $\begin{array}{c}\text { Convexa en ambas } \\
\text { caras. }\end{array}$ & $\begin{array}{c}\text { Convexa en ambas } \\
\text { caras. }\end{array}$ & $\begin{array}{c}\text { Convexa en } \\
\text { abax. }\end{array}$ \\
\hline & & Emp continuo. & Emp continuo. & Emp discontinuo. & Emp continuo \\
\hline & Vena media & $\begin{array}{l}1 \text { capa de co subepi en } \\
\text { ambas caras y } 1 \text { capa } \\
\text { de co que se continúa } \\
\text { con el haz en adax }\end{array}$ & $\begin{array}{c}1 \text { capa de co subepi en } \\
\text { ambas caras }\end{array}$ & $\begin{array}{c}2 \text { capas de co subepi } \\
\text { en adax y } 1 \text { capa en } \\
\text { abax }\end{array}$ & Sin datos \\
\hline \multirow{4}{*}{ 옴 } & Cutícula & $\begin{array}{c}\text { Ligeramente gruesa y } \\
\text { rugosa }\end{array}$ & Delgada y algo estriada & Delgada y lisa & Delgada y lisa \\
\hline & Cristales & Areniscas & Areniscas y drusas & Areniscas & Areniscas \\
\hline & \multirow[t]{2}{*}{$\begin{array}{l}\text { Tejidos } \\
\text { fundamentales }\end{array}$} & $\begin{array}{l}1 \text { capas de co subepi } \\
\text { en ambas caras }\end{array}$ & $\begin{array}{l}2 \text { capas de co subepi } \\
\text { en ambas caras }\end{array}$ & $\begin{array}{l}2 \text { capas de co subepi } \\
\text { en ambas caras }\end{array}$ & $\begin{array}{c}1 \text { capa de co } \\
\text { subepi en ambas } \\
\text { caras }\end{array}$ \\
\hline & & Sin fibras & Sin fibras & Sin fibras & Sin fibras \\
\hline
\end{tabular}

de oxalato en la lámina y/o el pecíolo. Por otra parte, la abundancia de los mismos varió de acuerdo al ambiente que las especies habitan, siendo mayor en aquellas de ambientes secos (C. cardenasii, $C$. chacoense y $C$. longidentatum).

No se hallaron diferencias significativas en el espesor de la lámina en corte transversal entre las especies que habitan ambientes secos (Chaqueña y de la Caatinga) y las que habitan ambientes húmedos (de las Yungas, Mata Atlántica, Pacífica y Paranaense). Por lo tanto, no se pudo comprobar que el aumento en el espesor de la lámina sea una estrategia que presentan las plantas de los ambientes xerofíticos (Dickison, 2000).

La cutícula también mostró variaciones en el espesor, encontrándose plantas que habitan en ambiente húmedo con cutícula delgada $(C$. flexuosum y $C$. cornutum) y con cutícula gruesa $(C$. baccatum). Sin embargo, es notable que en la lámina de todas las especies que habitan ambientes secos, la cutícula es considerablemente gruesa, coincidiendo con lo informado por Cutler (1987) para los xerófitos.

La proporción de tejido asimilador en la hoja de los xerófitos es generalmente mayor que en mesófitos debido a un incremento en el número de capas de células en empalizada (Fahn \& Cutler, 1992). En nuestro estudio todas las especies mostraron sólo una capa de células en empalizada; tampoco se observó que las especies que habitan en ambientes secos mostraran una relación parénquima en empalizada/parénquima esponjoso alta, ya que el primero, por su estructura, incrementa enormemente la superficie interna libre de la hoja, permitiendo mayor eficiencia en el intercambio de gases (Fahn \& Cutler, 1992). 


\section{V. Palchetti et al. - Anatomía foliar en Capsicum}

Tabla 7. Características cuantitativas epidérmicas para cada epidermis foliar de especies de Capsicum. Las variables se expresan como media \pm desvío estándar. Los valores cero simbolizan ausencia. Abreviaturas, abax: abaxial, adax: adaxial, Cél. Epi.: células epidérmicas, Tri. Egla.: tricomas eglandulares, Tri. Gla.: tricomas glandulares.

\begin{tabular}{|c|c|c|c|c|c|c|}
\hline Especie & $\begin{array}{l}\text { Cara } \\
\text { Foliar }\end{array}$ & $\begin{array}{l}\text { Densidad } \\
\text { Estomas }^{1}\end{array}$ & Densidad Cél. Epi. ${ }^{2}$ & $\begin{array}{c}\text { Índice } \\
\text { estomático }^{3}\end{array}$ & $\begin{array}{c}\text { Densidad Tri. } \\
\text { Egla. }\end{array}$ & $\begin{array}{c}\text { Densidad Tri. } \\
\text { Gla. }^{5}\end{array}$ \\
\hline \multirow{2}{*}{ C.baccatum } & abax & $237,87 \pm 58,3$ & $862,67 \pm 203,85$ & $21,62 \pm 1,71$ & $0,49 \pm 0,73$ & $2,83 \pm 2,13$ \\
\hline & adax & $11,71 \pm 10,54$ * & $696,27 \pm 159,55$ & $1,57 \pm 1,28^{a}$ & $1,35 \pm 1,17$ & $0,12 \pm 0,43$ * \\
\hline \multirow{2}{*}{ C.caatingae } & abax & $158,58 \pm 53,12$ & $560,71 \pm 140,36$ & $21,65 \pm 2,42$ & $27,55 \pm 23,09$ & 0 \\
\hline & $\operatorname{adax}$ & 0 & $476,09 \pm 125,76$ & 0 & $7,38 \pm 6$ & 0 \\
\hline \multirow{2}{*}{ C.cardenasii } & abax & $164 \pm 60,96$ & $880 \pm 72,27$ & $15,57 \pm 5,29$ & $0,74 \pm 1,48$ & $5,54 \pm 7,17$ \\
\hline & adax & $36,8 \pm 29,62^{a}$ & $736 \pm 209,89$ & $4,43 \pm 2,59^{a}$ & $0,74 \pm 1,48$ & $4,06 \pm 1,41$ \\
\hline \multirow{2}{*}{ C.chacoense } & abax & $196,95 \pm 33,42$ & $877,67 \pm 152,62$ & $18,49 \pm 3,04$ & $1,21 \pm 0,89$ & $0,54 \pm 1$ \\
\hline & adax & $38,98 \pm 38,92^{a}$ & $625,45 \pm 118,01^{a}$ & $5,12 \pm 4,49^{a}$ & $2,82 \pm 3,93$ & $0,13 \pm 0,45$ \\
\hline \multirow{2}{*}{ C.cornutum } & abax & $190,13 \pm 98,35$ & $638,67 \pm 228,31$ & $21,89 \pm 3,33$ & $7,51 \pm 2,78$ & $0,12 \pm 0,43$ \\
\hline & adax & 0 & $410,67 \pm 125,74^{*}$ & 0 & $4,80 \pm 3,78$ & $0,12 \pm 0,43$ \\
\hline \multirow{2}{*}{ C.eximium } & abax & $281,31 \pm 115,86$ & $1102,25 \pm 399,12$ & $20,08 \pm 3,62$ & $0,13 \pm 0,45$ & $13,29 \pm 11,77$ \\
\hline & adax & $11,35 \pm 20,71$ * & $863,42 \pm 347,34$ & $1,16 \pm 2,05$ * & $2,15 \pm 3,39$ * & $2,68 \pm 2,86$ * \\
\hline \multirow{2}{*}{ C.flexuosum } & abax & $193,07 \pm 85,26$ & $858,93 \pm 205,02$ & $17,85 \pm 4,14$ & $0,25 \pm 0,58$ & $0,25 \pm 0,58$ \\
\hline & adax & $4,53 \pm 8,68$ * & $551,20 \pm 125,56^{*}$ & $0,78 \pm 1,4$ * & $0,25 \pm 0,58$ & $1,11 \pm 1,43$ \\
\hline \multirow{2}{*}{ C.friburgense } & abax & $201,07 \pm 83$ & $697,33 \pm 154,64$ & $21,77 \pm 5,12$ & $0,13 \pm 0,45$ & $5,54 \pm 4,41$ \\
\hline & adax & $3,73 \pm 8,05$ * & $598,4 \pm 252,94$ * & $0,7 \pm 1,59$ * & $0,67 \pm 1,02$ & $0,37 \pm 0,67$ * \\
\hline \multirow{2}{*}{ C.geminifolium } & abax & $147,2 \pm 41,93$ & $901,6 \pm 273,69$ & $14,39 \pm 4,28$ & 0 & $4,06 \pm 2,05$ \\
\hline & adax & 0 & $510,8 \pm 181,36^{a}$ & 0 & 0 & $0,19 \pm 0,52$ * \\
\hline \multirow{2}{*}{ C.hunzikerianum } & abax & $231,31 \pm 58,42$ & $779,43 \pm 140,09$ & $22,57 \pm 1,94$ & $28,04 \pm 21,05$ & $4,22 \pm 3$ \\
\hline & adax & $4,11 \pm 7,55$ * & $498,74 \pm 54,91$ * & $0,77 \pm 1,45^{a}$ & $2,11 \pm 1,67^{*}$ & $2,32 \pm 1,67$ \\
\hline \multirow{2}{*}{ C.longidentatum } & abax & $128 \pm 55,1$ & $558,58 \pm 90,53$ & $17,93 \pm 4,12$ & $27,72 \pm 29,42$ & $3,77 \pm 3,62$ \\
\hline & adax & $4,27 \pm 5,77^{*}$ & $338,84 \pm 39,21$ * & $1,18 \pm 1,56$ * & $21,98 \pm 23,4$ & $0,16 \pm 0,49$ * \\
\hline \multirow{2}{*}{ C.recurvatum } & abax & $296,27 \pm 69,26$ & $984,8 \pm 229,81$ & $23,13 \pm 1,95$ & $2,83 \pm 4,34$ & $4,68 \pm 2,87$ \\
\hline & adax & $27,47 \pm 16,79$ * & $571,47 \pm 79,3$ * & $4,52 \pm 2,97^{a}$ & $1,23 \pm 1,52$ & $0,74 \pm 1,18$ * \\
\hline \multirow{2}{*}{ C.villosum } & abax & $216 \pm 71,84$ & $722,88 \pm 111,67$ & $22,4 \pm 4,05$ & $1,77 \pm 2,39$ & $3,69 \pm 1,87$ \\
\hline & adax & $4,48 \pm 8,69$ * & $485,76 \pm 33,45$ * & $0,84 \pm 1,63$ * & $1,33 \pm 1,47$ & $0,74 \pm 1,44$ * \\
\hline
\end{tabular}

1- estomas $/ \mathrm{mm}^{2} ;$ 2- células epidérmicas $/ \mathrm{mm}^{2}$

3- índice estomático $=($ densidad estomática/densidad estomática + densidad de cél. epi. $) .100$

4- tricomas eglandulares $/ \mathrm{mm}^{2} ;$ 5- tricomas glandulares $/ \mathrm{mm}^{2}$

*- diferencias significativas entre caras foliares de una especie mediante test no paramétrico Kruskal Wallis $(p<0,05)$

a- diferencias significativas entre caras foliares de una especie mediante test paramétrico $(p<0,05)$

Según Smith et al. (1998), la orientación de las hojas y su exposición a la luz incide en las características anatómicas. Sería, por lo tanto, interesante probar a campo esto último ya que la orientación de la hoja se considera otro factor influyente en la anatomía. Por otra parte, tampoco se encontró una relación entre el tamaño de los espacios intercelulares y el ambiente dado la alta variabilidad que se manifestó en este carácter.

La aparición de cristales, resinas, aceites y taninos en las células de la hoja ha sido considerada de valor adaptativo a condiciones áridas (Fahn \& Cutler, 1992). Por lo tanto, la abundancia de areniscas cristalinas y drusas en $C$. longidentatum y C. cardenasii, especies de ambientes secos, y la probable presencia de taninos en $C$. caatingae estarían en concordancia con lo antedicho. No fue observado desarrollo adicional de tejido esclerenquimático, sólo C. geminifolium que habita en ambiente húmedo presentó fibras asociadas al floema. 
Tabla 8. Comparación entre las epidermis abaxial y adaxial de 13 especies de Capsicum para la variable densidad de estomas. Los valores indicados son las medias. Los valores cero simbolizan ausencia. Diferentes letras representan diferencias significativas según Kruskal Wallis $(p<0,05)$.

\begin{tabular}{|c|c|c|c|c|c|c|c|c|c|c|c|}
\hline \multicolumn{7}{|c|}{ EPIDERMIS ABAXIAL } & \multicolumn{5}{|c|}{ EPIDERMIS ADAXIAL } \\
\hline Especie & & & & & & & Especie & & & & \\
\hline C. Iongidentatum & $128^{*}$ & $A$ & & & & & C. geminifolium & $0^{*}$ & $A$ & & \\
\hline C. geminifolium & $147,2^{*}$ & A & B & & & & C. cornutum & $0^{*}$ & A & & \\
\hline C. caatingae & $158,6^{*}$ & A & B & C & & & C. caatingae & $0^{*}$ & A & & \\
\hline C. cardenasii & $164^{*}$ & $A$ & B & $\mathrm{C}$ & D & & C. friburgense & $3,7^{*}$ & $A$ & $\mathrm{~B}$ & \\
\hline C. cornutum & $190,1^{*}$ & A & B & C & D & & C. hunzikerianum & $4,1^{*}$ & $A$ & $\mathrm{~B}$ & \\
\hline C. flexuosum & $193,1^{*}$ & A & B & $\mathrm{C}$ & $\mathrm{D}$ & & C. villosum & $4,5^{\star}$ & A & $\mathrm{B}$ & \\
\hline C. friburgense & $201,1^{*}$ & & B & C & D & & C. flexuosum & $4,5^{*}$ & $A$ & $\mathrm{~B}$ & \\
\hline C. chacoense & $197^{*}$ & & B & C & D & & C. eximium & $11,4^{*}$ & A & B & \\
\hline C. villosum & $216^{*}$ & & & C & D & $E$ & C. longidentatum & $4,3^{*}$ & $A$ & $\mathrm{~B}$ & \\
\hline C. hunzikerianum & $231,3^{*}$ & & & & D & $E$ & C. baccatum & $11,7^{*}$ & & $\mathrm{~B}$ & C \\
\hline C. baccatum & $237,9^{*}$ & & & & D & $E$ & C. chacoense & $39^{\star}$ & & & C \\
\hline C. eximium & $281,3^{*}$ & & & & $\mathrm{D}$ & $\bar{E}$ & C. recurvatum & $27,5^{*}$ & & & C \\
\hline C. recurvatum & $296,3^{*}$ & & & & & $E$ & C. cardenasii & $36,8^{*}$ & & & C \\
\hline
\end{tabular}

* valor medio

Tabla 9. Comparación entre las epidermis adaxial y abaxial de 13 especies de Capsicum para la variable densidad de tricomas eglandulares. Los valores indicados son las medias. Los valores cero simbolizan ausencia. Diferentes letras representan diferencias significativas según Kruskal Wallis $(p<0,05)$.

\begin{tabular}{|c|c|c|c|c|c|c|c|c|c|c|c|c|c|}
\hline \multicolumn{6}{|c|}{ EPIDERMIS ABAXIAL } & \multicolumn{8}{|c|}{ EPIDERMIS ADAXIAL } \\
\hline Especie & & & & & & Especie & & & & & & & \\
\hline C. geminifolium & $0^{*}$ & A & & & & C. geminifolium & $0^{*}$ & A & & & & & \\
\hline C. friburgense & $0,1^{*}$ & A & & & & C. flexuosum & $0,3^{*}$ & A & B & & & & \\
\hline C. eximium & $0,1^{*}$ & A & & & & C. cardenasii & $0,7^{*}$ & A & B & C & & & \\
\hline C. flexuosum & $0,3^{*}$ & A & & & & C. friburgense & $0,7^{*}$ & A & $B$ & C & & & \\
\hline C. cardenasii & $0,7^{*}$ & A & & & & C. recurvatum & $1,2^{*}$ & A & B & C & & & \\
\hline C. baccatum & $0,5^{*}$ & A & & & & C. eximium & $2,2^{*}$ & A & $B$ & C & $\mathrm{D}$ & & \\
\hline C. recurvatum & $2,8^{*}$ & A & & & & C. villosum & $1,3^{*}$ & A & $B$ & C & $\mathrm{D}$ & & \\
\hline C. villosum & $1,8^{*}$ & A & B & & & C. baccatum & $1,4^{*}$ & & B & C & D & & \\
\hline C. chacoense & $1,2^{*}$ & A & $\mathrm{B}$ & C & & C. chacoense & $2,8^{*}$ & & B & C & $\mathrm{D}$ & $\mathrm{E}$ & \\
\hline C. caatingae & $27,6^{*}$ & & B & C & $\mathrm{D}$ & C. hunzikerianum & $2,1^{*}$ & & & C & D & $\mathrm{E}$ & \\
\hline C. longidentatum & $27,7^{*}$ & & & $\mathrm{C}$ & D & C. cornutum & $4,8^{*}$ & & & & $\mathrm{D}$ & $\mathrm{E}$ & $\mathrm{F}$ \\
\hline C. cornutum & $7,5^{\star}$ & & & & $\mathrm{D}$ & C. caatingae & $7,4^{*}$ & & & & & $E$ & $\mathrm{~F}$ \\
\hline C. hunzikerianum & $28^{*}$ & & & & $\mathrm{D}$ & C. Iongidentatum & $22^{*}$ & & & & & & $\mathrm{~F}$ \\
\hline
\end{tabular}

* valor medio

Conforme a los resultados obtenidos del análisis de la estructura de la hoja en especies de Capsicum, es interesante destacar que fueron pocos los caracteres que se correlacionaron directamente con el hábitat, no encontrándose una diferencia notable entre las plantas que crecen en las distintas Provincias Biogeográficas caracterizadas por su ambiente húmedo de aquellas de ambientes secos. Cutler (1987) y Laskowski (1996) indicaron que no siempre las adaptaciones son evidentes a nivel morfológico. En tal sentido, pueden existir adaptaciones fisiológicas que han evolucionado hacia formas vegetales aptas para crecer ante situaciones de déficit hídrico y altas temperaturas.
En referencia al valor de los caracteres a nivel taxonómico, encontramos particularidades que sólo se presentan en algunas especies tales como: hojas hipostomáticas en $C$. caatingae, $C$. cornutum y $C$. geminifolium; ausencia de tricomas glandulares en $C$. caatingae y eglandulares simples en $C$. geminifolium; presencia de tricomas eglandulares ramificados en $C$. longidentatum; probable presencia de taninos en $C$. caatingae; haz vascular central rodeado por fibras en C. geminifolium; ausencia de cristales en $C$. flexuosum; abundancia de cristales en C. cardenasii y C. longidentatum. Estas particularidades, sumadas a otras más generales, se muestran en la siguiente clave: 


\section{V. Palchetti et al. - Anatomía foliar en Capsicum}

1. Hojas con denso indumento de tricomas eglandulares ramificados

C. longidentatum

1 '. Hojas con indumento variable de tricomas eglandulares simples o de tricomas glandulares

2. Hojas hipostomáticas

3. Vena media de la lámina sin colénquima subepidérmico y haz vascular rodeado de fibras; haz vascular del pecíolo en forma de U

3'. Vena media de la lámina con colénquima subepidérmico y haz vascular rodeado de parénquima; haz vascular del pecíolo en forma de arco

4. Células epidérmicas y del parénquima en empalizada sin contenido denso citoplasmático; parénquima en empalizada continuo en la vena media

C. cornutum

4'. Células epidérmicas y del parénquima en empalizada con marcado contenido denso citoplasmático; mesofilo con areniscas cristalinas; parénquima en empalizada discontinuo en la vena media

C. caatingae

2. Hojas anfistomáticas

3. Estomas exclusivamente anisocíticos en la cara adaxial y abaxial; abundantes cristales: drusas en el mesofilo y areniscas cristalinas alrededor del haz vascular de la lámina y pecíolo

C. cardenasii

3'. Estomas por lo general anomocíticos o hemiparacíticos (salvo en C. recurvatum y C. villosum, donde también aparecen estomas anisocíticos); cristales frecuentes (drusas y/o areniscas cristalinas) en lámina y pecíolo, o ausentes

4. Cristales totalmente ausentes en la lámina

5. Pecíolos sin cristales de ningún tipo; estomas anisocíticos ausentes; colénquima subepidérmico ausente en la vena media de la lámina

C. flexuosum

5'. Pecíolos con frecuentes areniscas cristalinas; estomas anisocíticos entremezclados con anomocíticos; colénquima subepidérmico distribuido en dos capas en la superficie abaxial de la vena media de la lámina

4'. Areniscas cristalinas y drusas o sólo drusas presentes en la lámina

C. recurvatum

5. Lámina sólo con drusas; estomas anisocíticos entremezclados con anomocíticos

5'. Lámina con drusas y areniscas cristalinas simultáneamente; estomas anomocíticos y hemiparacíticos, los anisocíticos ausentes

6. Colénquima ausente en pecíolo y hasta con 4 capas subepidérmicas en la superficie adaxial de la vena media de la lámina; parénquima en empalizada discontinuo sobre la vena media

C. friburgense

6'. Colénquima presente en pecíolo (1-3 capas) y ausente o hasta con 2 capas subepidérmicas en la superficie adaxial de la vena media de la lámina; parénquima en empalizada continuo sobre la vena media

7. Parénquima esponjoso distribuido en 2-3 capas en el lámina; relación parénquima empalizada/parénquima esponjoso $=1 / 1$

8. Colénquima subepidérmico distribuido en dos capas en la superficie adaxial de la vena media de la lámina, y en tres capas en la superficie adaxial del pecíolo

C. hunzikerianum

8'. Colénquima subepidérmico distribuido en una capa en ambas caras de la vena media de la lámina, y en dos capas en ambas caras del pecíolo

C. eximium

7'. Parénquima esponjoso distribuido en 5-6 capas en el lámina; relación parénquima empalizada/parénquima esponjoso $=3 / 4$

8. Células de la epidermis adaxial con paredes subrectas

8'. Células de la epidermis adaxial con paredes sinuosas

C. baccatum

C. chacoense 
Tabla 10. Comparación entre las epidermis adaxial y abaxial de 13 especies de Capsicum para la variable densidad de tricomas glandulares. Los valores indicados son las medias. Los valores cero simbolizan ausencia. Diferentes letras representan diferencias significativas según Kruskal Wallis $(p<0,05)$.

\begin{tabular}{|c|c|c|c|c|c|c|c|c|c|c|}
\hline \multicolumn{5}{|c|}{ EPIDERMIS ABAXIAL } & \multicolumn{6}{|c|}{ EPIDERMIS ADAXIAL } \\
\hline Especies & & & & & Especies & & & & & \\
\hline C. caatingae & $0^{*}$ & A & & & C. caatingae & $0^{*}$ & A & & & \\
\hline C. cornutum & $0,1^{*}$ & A & & & C. cornutum & $0,1^{*}$ & $A$ & & & \\
\hline C. flexuosum & $0,3^{*}$ & A & & & C. baccatum & $0,1^{*}$ & $A$ & & & \\
\hline C. chacoense & $0,5^{*}$ & A & & & C. chacoense & $0,1^{*}$ & A & & & \\
\hline C. baccatum & $2,8^{*}$ & & B & & C. Iongidentatum & $0,2^{*}$ & A & & & \\
\hline C. Iongidentatum & $3,8^{*}$ & & B & C & C. geminifolium & $0,2^{*}$ & A & & & \\
\hline C. cardenasii & $5,5^{*}$ & & B & C & C. friburgense & $0,4^{*}$ & A & & & \\
\hline C. villosum & $3,7^{*}$ & & B & C & C. villosum & $0,7^{*}$ & A & B & & \\
\hline C. hunzikerianum & $4,2^{*}$ & & B & C & C. recurvatum & $0,7^{*}$ & A & B & & \\
\hline C. geminifolium & $4,1^{*}$ & & B & C & C. flexuosum & $1,1^{*}$ & $A$ & B & C & \\
\hline C. recurvatum & $4,7^{*}$ & & B & C & C. eximium & $2,7^{*}$ & & B & C & $\mathrm{D}$ \\
\hline C. friburgense & $5,5^{*}$ & & B & C & C. hunzikerianum & $2,3^{*}$ & & & C & $\mathrm{D}$ \\
\hline C. eximium & $13,3^{*}$ & & & C & C. cardenasii & $4,1^{*}$ & & & & $\mathrm{D}$ \\
\hline
\end{tabular}

* valor medio

Por último, habría que analizar si las similitudes encontradas en los valores de las variables epidérmicas y ciertas características de la estructura interna de las hojas entre determinadas especies, son coincidentes principalmente con un agrupamiento según sus relaciones filogenéticas más que con las condiciones ambientales donde habitan. Estos caracteres podrán ser valorados en tal sentido, ampliando el estudio hacia las restantes especies de Capsicum y realizando un mapeo de los caracteres sobre una filogenia molecular del género, estudios que actualmente está desarrollando nuestro grupo de trabajo.

\section{AGRADECIMIENTOS}

Los autores agradecen a los revisores anónimos por las valiosas sugerencias para mejorar el manuscrito; a las instituciones que financiaron este estudio, Secretaría de Ciencia y Tecnología de la Universidad Nacional de Córdoba (SECYT, UNC), Consejo Nacional de Investigaciones Científicas y Técnicas (CONICET) y Agencia de Promoción Científica y Técnica y Ministerio de Ciencia y Tecnología de la Provincia de Córdoba.

\section{Bibliografía}

BARBOZA, G. E. \& L. BIANCHETTI. 2005. Three new species of Capsicum (Solanaceae) and a key to the wild species from Brazil. Syst. Bot. 30: 863-871.

BARBOZA, G. E., M. F. AGRA, M. V. ROMERO, M. A. SCALDAFERRO \& E. A. MOSCONE. 2011. New Endemic Species of Capsicum (Solanaceae) from the Brazilian Caatinga: Comparison with the Recircumscribed C. parvifolium. Syst. Bot. 36: 768-781.

BARCELOUX, D. G. 2008. Pepper and capsaicin (Capsicum and Piper species). In: BARCELOUX, D. G. (ed.), Medical toxicology of natural substances: Foods, fungi, medicinal herbs, toxic plants, and venomous animals, pp. 71-76. John Wiley \& Sons, Inc, New Jersey.

BESSIS, J. \& M. GUYOT. 1979. An attempt to use stomatal characters in systematic and phylogenetic studies of the Solanaceae. In: J. G. HAWKES, R. N. LESTER \& A. D. SKELDING (eds.), The biology and taxonomy of the Solanaceae. Linn. Soc. Symp. Ser. 7: 321-326. Academic Press, London.

BUCKENHÜSKES, H. J. 2003. Current requirements on paprika powder for food industry. In: DE, A. K. (ed.), Capsicum: The genus Capsicum, pp. 223-230. Taylor and Francis, London and New York.

CABRERA, A. L. \& A. WILLINK. 1980. Biogeografía de América Latina. Serie de Biología. Monograph 13: 1-122. Secretaría General de la OEA, Washington, D. C. 
CONN, H. J., M. A. DARROW \& V. M. EMMEL. 1960. Staining procedures I-XII. Williams and Wiltings, Baltimore, Maryland.

COSA, M. T. 1991. Estudio morfoanatómico de órganos vegetativos en Cestroideae (Solanaceae). I: Tribu Nicotianeae. Kurtziana 21: 111-152.

COSA, M. T. 1993. Estudio morfoanatómico de órganos vegetativos en Cestroideae (Solanaceae). II: Tribu Salpiglossideae. Kurtziana 22: 47-72.

COSA, M. T., M. HADID, N. DOTTORI \& G. BRUNO. 2002. Anatomía de órganos vegetativos en Solanum palinacanthum, $S$. sisymbrifolium y $S$. euacanthum (Solanaceae). Anales Inst. Biol Univ. Nac. Autón. México, Ser. Bot. 73: 27-38.

CUTLER, D. F. 1987. Anatomía vegetal aplicada. Ed. Mosaico, Argentina.

D'AMBROGIO DE ARGÜESO, A. M. 1986. Manual de Técnicas en Histología Vegetal. Ed. Hemisferio Sur, Argentina.

DELBÓN, N., M. T. COSA, N. DOTTORI \& L. STIEFKENS. 2007. Análisis de los caracteres epidérmicos en Flourensia campestris y $F$. oolepis (Asteraceae). Bol. Soc. Argent. Bot. 42: 245-250.

DI RIENZO, J., F. CASANOVES, M. BALZARINI, L. GONZALEZ, M. TABLADA \& C. ROBLEDO. 2011. InfoStat Versión 2011. Grupo InfoStat, FCA, Universidad Nacional de Córdoba, Argentina. Disponible en: http://www.infostat.com.ar/

DICKISON, W. C. 2000. Integrative Plant Anatomy. Harcourt/Academic Press, San Diego.

FAHN, A. 1990. Plant Anatomy. Pergamon Press, Oxford.

FAHN, A. \& D. F. CUTLER. 1992. Xerophytes. Handbuchder Pflanzenantomie. Gebruder Borntraeger, Berlin.

FUTUYMA, D. J. 2009. Evolution. 2nd ed. Sinauer Associates, Inc. Sunderland, Mussachusetts.

GIULIETTI, A. M., R. M. HARLEY, L. P. DE QUEIROZ, M. R. V. BARBOSA, A. L. BOCAGE-NETA \& M. A. FIGUEIREDO. 2002. Espécies endêmicas da Caatinga. In: SAMPAIO, E. V., A. M. GIULIETTI, J. VIRGINIO \& C. F. GAMARRA-ROJAS (eds.), Vegetação \& Flora da Caatinga, pp. 103-108. Associação Plantas do Nordeste, Recife.

HUNZIKER, A. T. 2001. The genera of Solanaceae. A.R.G. Gantner Verlag K.-G., Ruggell, Liechtenstein.
KARATELA, Y. \& L. S. GILL. 1986. Observation on the developmental studies of stomatal differentiation in the epidermis of Solanaceae. Feddes Repert. 97: 303-311.

LASKOWSKI, L. 1996. Selección, propagación y anatomía del semeruco. Tesis de grado. Posgrado de Horticultura. Universidad Centroccidental Lisandro Alvarado, Venezuela.

LISCOVSKY, I. J. \& M. T. COSA. 2005. Anatomía comparativa de hoja y tallo en los representantes de Cestreae G. Don (Solanaceae) de Argentina. Gayana Bot. 62: 33-43.

LLERAS, E. 1997. Caatinga of North-Eastern Brazil. In DAVIS S. D., V. H. HEYWOOD, O. HERRERA-MACBRYDE, J. VILLALOBOS \& A. HAMILTON (eds.), Centres of Plant Diversity: A guide and strategy for their conservation. Vol. 3: The Americas. IUCN Publications Unit, Cambridge, England. Disponible en: http://botany.si.edu/projects/cpd/ [Acceso: 12 February 2014].

MENTZ, L. A., P. L. DE OLIVEIRA \& M. V. DA SILVA. 2000. Tipologia dos tricomas das espécies do gênero Solanum (Solanaceae) na Região Sul do Brasil. Iheringia, Sér. Bot. 54: 75-106.

METCALFE, C.R. \& L. CHALK. 1957. Anatomy of the Dicotyledons. Vol. 2. Clarendon press, Oxford.

METCALFE, C.R. \& L. CHALK. 1983. Anatomy of the Dicotyledons. 2nd ed. Vol. 2. Wood structure and conclusion of the general introduction. Clarendon Press, Oxford.

MOSCONE, E. A., M. A. SCALDAFERRO, M. GABRIELE, N, M. CECCHINI, Y. SÁNCHEZ GARCÍA, R. JARRET, J. R. DAVIÑA, D. A. DUCASSE, G. E. BARBOZA \& F. EHRENDORFER. 2007. The evolution in chili peppers (CapsicumSolanaceae), a cytogenetic perspective. Acta Hort. 745: 137-170.

OLMSTEAD, R., L. BOHS, H. A. MIGID, E. SANTIAGO-VALENTÍN, V. F. GARCÍA \& S. M. COLLIER. 2008. A molecular phylogeny of the Solanaceae. Taxon 57: 1159-1181.

QUEIROZ, L. P. 2006. The Brazilian caatinga: phytogeographical patterns inferred from distribution data of the Leguminosae. In: PENNINGTON, R. T., G. P. LEWIS, \& J. A. RATTER (eds.), Neotropical dry forests and 
Bol. Soc. Argent. Bot. 49 (3) 2014

savannas, pp. 113-149. Royal Botanical Garden, Edinburgh.

RIGONATTO, O., N. DOTTORI \& M. T. COSA. 2005. Anatomía de órganos vegetativos en Solanum argentinum (Solanaceae). Kurtziana 31: 21-28.

ROE, K. E. 1972. A revision of Solanum section Brevantherum (Solanaceae). Brittonia 24(3): 239-278.

SÄRKINEN, T., R.T. PENNINGTON, M. LAVIN, M. F. SIMON \& C. E. HUGHES. 2013. Evolutionary islands in the Andes: persistence and isolation explain high endemism in Andean dry tropical forests. $J$. Biogeogr. 39: 884-900.

SCHEFFÉ, H. 1959. The Analysis of Variance. Ed. John Wiley, New York.

SEITHE, A. 1979. Hair types as taxonomic characters in Solanum. In: J. G. HAWKES, R. N. LESTER \& A. D. SKELDING (eds.), The biology and taxonomy of the Solanaceae. Linn. Soc. Symp. Ser. 7: 307-319. Academic Press, London.

SMITH, W. K., D. T. BELL \& K. A. SHEPHERD. 1998. Associations between leaf structure, orientation, and sunlight exposure in five Western Australian communities. Amer. J. Bot. 85: 56-63.

SOKAL, R. R. \& F. J. ROHLF. 1981. The Principles and Practice of Statistic in
Biological Research. Ed. Freeman and Co., San Francisco.

STACE, C. A. 1965. Cuticular studies as an aid to plant taxonomy. Bull. Brit. Mus. (Nat. Hist.), Bot. 4: 1-78.

THAKUR, P. S. 1990. Different physiological responses of tomato (Lycopersicon esculentum Mill.) cultivars to drought. Acta Physiol. Pl. 12: 175-182.

TORAL, M., A. MANRÍQUEZ, R. NAVARROCERRILLO, D. TERSI \& P. NAULIN. 2010. Características de los estomas, densidad e índice estomático en secuoya (Sequoia sempervirens) y su variación en diferentes plantaciones de Chile. Bosque 31: 157-164.

WOOD, J. G. 1934. The physiology of xerophytism in Australian plants. The stomatal frequencies, transpiration and osmotic pressures of sclerophyll and tomentosesucculent leaved plants. J. Ecol. 22: 69-87.

Recibido el 11 de marzo de 2013, aceptado el 22 de mayo de 2014. 